\title{
A Skeptical Theory of Inheritance in Nonmonotonic Semantic Networks
}

\author{
John F. Horty \\ Philosophy Department and Institute for Advanced Computer \\ Studies, University of Maryland, College Park, MD 20742, \\ USA
}

Richmond H. Thomason

Intelligent Systems Program, University of Pittsburgh, Pittsburgh, PA 15260, USA

\author{
David S. Touretzky \\ Computer Science Department, Carnegie-Mellon University, \\ Pittsburgh, PA 15213, USA
}

\begin{abstract}
This paper describes a new approach to inheritance reasoning in semantic networks allowing for multiple inheritance with exceptions. The approach leads to an analysis of defeasible inheritance which is both well-defined and intuitively attractive: it yields unambiguous results applied to any acyclic semantic network, and the results conform to our intuitions in those cases in which the intuitions themselves are firm and unambiguous. Since the definition provided here is based on an alternative, skeptical view of inheritance reasoning, however, it does not always agree with previous definitions when it is applied to nets about which our intuitions are unsettled, or in which different reasoning strategies could naturally be expected to yield distinct results. After exploring certain features of the definition presented here, we describe also a hybrid (parallel-serial) algorithm that implements the definition in a parallel marker-passing architecture.
\end{abstract}

\section{Introduction}

This paper describes a new approach to inheritance reasoning in semantic networks allowing for multiple inheritance with exceptions. Like the previous approaches of Touretzky [22] and Etherington [4], but unlike many other approaches, such as those of Roberts and Goldstein [19] or Fahlman [5], the approach presented here leads to an analysis of defeasible inheritance which is both well-defined and intuitively attractive: it yields unambiguous results 0004-3702/90/\$3.50 (C) 1990, Elsevier Science Publishers B.V. (North-Holland) 
applied to any acyclic semantic network, and the results conform to our intuitions in those cases in which our intuitions themselves are firm and unambiguous. Since the definition provided here is based on an alternative, skeptical view of inheritance reasoning, however, it does not always agree with these previous definitions when it is applied to nets about which our intuitions are unsettled, or in which different reasoning strategies could naturally be expected to yield distinct results.

The paper is organized as follows. In Section 2, after setting out our notation and basic terminology, we sketch a view of the general nature of inheritance reasoning in nonmonotonic networks, drawing upon a loose analogy with ordinary deductive reasoning. In Section 3, we isolate the principles underlying our particular approach to inheritance. These principles are then organized into a rigorous definition in Section 4 , and the resulting definition is examined in more detail in Section 5. An actual inheritance reasoner based on the definition presented here has been implemented in COMMON LISP; the program, along with some sample inputs and runs, can be found in Horty et al. [11]. Of more theoretical interest, we describe in Section 6 of this paper a hybrid (parallel-serial) algorithm that implements our inheritance definition in a parallel marker-passing architecture.

Although we offer a few scattered remarks, we do not attempt to provide any systematic comparison between the approach to nonmonotonic inheritance presented here and other approaches described elsewhere in the literature. Very briefly, our approach differs from many of those based on nonmonotonic logics, such as that of Etherington [4], in allowing us to give a uniform meaning to each link of a network. We are not forced to devise different logical representations for the same link when it occurs in different networks; and indeed, we do not translate the links from these networks into an intermediate logical formalism at all, but set out our theory directly, in terms of the network language itself. In this respect, the approach taken here agrees with the previous approach of Touretzky [22]. However, it differs both from Touretzky's approach and from that of Etherington in this way: while both of these theories can associate a number of different extensions with a single network, the skeptical theory described in this paper always leads to a unique extension. Because it allows us to avoid the complexities of dealing with multiple extensions, this skeptical approach may prove to be more practical in some applications.

\section{Basic Concepts}

\subsection{Notation}

Letters from the beginning of the alphabet $(a, b, c)$ will represent objects, and letters from the middle of the alphabet $(p, q, r)$ will represent kinds of objects. 
Letters from the end of the alphabet $(u, v, w, x, y, z)$ will range over both objects and kinds.

An assertion will have the form $x \rightarrow y$ or $x \nrightarrow y$, where $y$ is a kind. If $x$ is an object, such an assertion is to be interpreted as an ordinary atomic statement: $a \rightarrow p$ and $b \nrightarrow p$, for instance, are analogous to $P a$ and $\neg P b$ in logic; they might represent statements like "Tweety is a bird" and "Jumbo isn't a bird." If $x$ is a kind, these assertions are to be interpreted as generic statements: $p \rightarrow q$ and $r \nrightarrow q$, for example, might represent the statements "Birds fly" and "Mammals don't fly." There is nothing in ordinary logic very close in meaning to generic statements like these. In particular, "Birds fly" cannot be interpreted through a universally quantified formula of the form $\forall x[P x \supset Q x]$, and "Mammals don't fly" does not mean anything like $\forall x[R x \supset \neg Q x]$, since the generic statements can be true even in the face of exceptions to the universal claims - a bird that cannot fly, for example. ${ }^{1}$ We describe a pair of assertions having the form $x \rightarrow y$ and $x \rightarrow y$ as conflicting assertions. Note that the conflicting assertions include not only logically contradictory pairs, like "Tweety is a bird" and "Tweety isn't a bird," but also the kind of conflicts exhibited by pairs of generic statements such as "Birds fly" and "Birds don't fly.",

Capital Greek letters will represent networks, where a network consists of a set $I$ of individuals and a set $K$ of kinds, together with a set of positive links and a set of negative links, both finite subsets of $(I \times K) \cup(K \times K)$. We identify the positive and negative links in a network with our positive and negative assertions.

Lower case Greek letters will range over sequences of links, among which we single out for special consideration the paths, defined inductively as follows: each assertion is a path; and if $\sigma \rightarrow p$ is a path, then both $\sigma \rightarrow p \rightarrow q$ and $\sigma \rightarrow p \nrightarrow q$ are paths. As this notation indicates, paths are special kinds of link sequences-joined, in the sense that the end node of any link in a path is identical with the initial node of the next link. It follows from their definition that paths are subject also to two further constraints. First, a negative link can occur in a path, if at all, only at the very end: $a \rightarrow p \rightarrow q$ is a path, but $a \nrightarrow p \rightarrow q$ isn't. Second, an individual can occur only as the initial node of a path: $p \rightarrow a \rightarrow q$ isn't a path.

Paths will be said to enable assertions, or statements, much in the way that proofs enable their conclusions: a path of the form $x \rightarrow \sigma \rightarrow y$ is said to enable the assertion $x \rightarrow y$, and likewise, a path of the form $x \rightarrow \sigma \nrightarrow y$ is said to enable the assertion $x \rightarrow y$. As this suggests, it is often natural to understand a path-like a proof-as representing a particular chain of reasoning behind the assertion it enables. The path $a \rightarrow p \rightarrow q$, for example, enables the assertion

\footnotetext{
'For detailed argumentation on this point, with supporting linguistic evidence, see Carlson [3]

2 Intuitively, these pairs are inconsistent. But whether the second is the negation of the first, and whether they are logically inconsistent, are issues that would have to be settled by a logic of the generic plural. Unfortunately, there is as yet no such logic.
} 
"Tweety flies," while representing an argument like "Tweety flies because he is a bird and birds fly." We describe a pair of paths as conflicting paths if they enable conflicting assertions.

\subsection{Inheritance}

Since we identify the links in a net with assertions, a net can be viewed as a set of hypotheses, or axioms. Let us say, informally for a moment, that an assertion $A$ is supported by a net $\Gamma$ if we can reasonably conclude that $A$ is true whenever all the links in $\Gamma$ are true-if the information contained in $\Gamma$ would naturally lead to the conclusion that $A$. Our object here is to explicate this informal idea. We want to know exactly what we can conclude from a given net; so we need to provide a formal account of the conditions under which a net $\Gamma$ supports an assertion $A$.

In the context of ordinary deductive logic, we often find ourselves in a similar situation, when we want to know what statements are deducible from a given set of hypotheses. In that context, it is a common practice to approach the question in a roundabout way. Instead of defining the relation of deducibility directly, one first characterizes the deductions-sequences of statements representing certain kinds of arguments, or chains of reasoning-and then defines a statement as deducible from a set of hypotheses if those hypotheses permit a deduction of that statement.

Of course, the process of drawing conclusions from a set of defeasible hypotheses through inheritance reasoning is quite different from the process of drawing conclusions from through deduction. Inheritance reasoning doesn't depend on the interplay of connectives, for example, since there aren't really any connectives, to speak of, in our semantic nets (the symbols $\rightarrow$ and $\rightarrow$ are not connectives, since they apply to individuals and kind terms rather than sentences, and they do not nest); and even some of the connective-free structural rules governing classical deducibility, such as weakening, fail to hold for nonmonotonic inheritance. Still, we find it helpful in the case of inheritance to follow a similar kind of roundabout strategy in describing the consequences of a set of hypotheses. Instead of trying to specify directly the statements supported by a given net, we first characterize the arguments or chains of reasoning-represented, now, by paths-that are permitted by a net. As in the case of ordinary deducibility, this relation between sets of hypotheses and the chains of reasoning they permit is really the central idea; and it will be the primary focus of our attention. Once we have identified the paths that a net permits, it is natural to define the statements supported by a net by stipulating that a net supports a statement just in case it permits a path enabling that statement.

We refer to the entire set of statements a net supports as the theory of the net, and to the entire set of paths it permits as its extension. 


\section{Motivation}

In this section we examine several simple examples of nets and the paths they should permit, in order to illustrate the principles underlying our general characterization of the permission relation, which is then presented in Section 4.

\subsection{Forward chaining}

Consider, first, the simplest kind of case imaginable, a linear net $\Gamma_{1}$ (Fig. 1). Just to fix an interpretation, let $a=$ Tweety, $p=$ Canaries, $q=$ Birds, and $r=$ Flying Things. $\Gamma_{1}$ explicitly contains the information, then, that Tweety is a canary, that canaries are birds, and that birds fly. Now given just this information, we would certainly want to allow a chain of reasoning along the lines of "Since Tweety is a canary, a kind of bird, and birds fly, Tweety flies"-so we want the net $\Gamma_{1}$ to permit the compound path $a \rightarrow p \rightarrow q \rightarrow r$, representing this argument. In just the same way, we want the net $\Gamma_{2}$ (Fig. 2), with $b=$ Jumbo, $s=$ Royal Elephants, $t=$ Elephants, and $u=$ Flying Things, to permit the path $b \rightarrow s \rightarrow t \rightarrow \rightarrow u$, which represents an argument something like "Jumbo is a royal elephant, a kind of elephant, and elephants don't fly; so Jumbo doesn't fly."

These examples illustrate some of the compound reasoning paths that can be constructed by assembling the direct links contained in a net, but they don't yet tell us, when we think of the construction as proceeding inductively, how these paths are to be assembled. There are, of course, two natural options for assembling compound paths from direct links: roughly, top-down and bottomup. Most treatments of inheritance reasoning-including those of Roberts and Goldstein [19], Fahlman [5], and Touretzky [22]-presume the top-down approach. They are guided, more or less explicitly, by a picture of inheritance according to which properties are imagined to flow downward through the semantic net, from more general to more specific kinds and then finally to individuals, unless the flow is interrupted, somehow, by an exception. Formally, this "property flow" picture leads to the construction of compound permit-

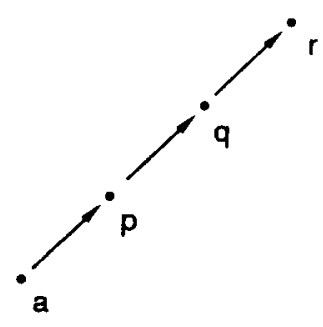

Fig. 1. $\Gamma_{1}$.

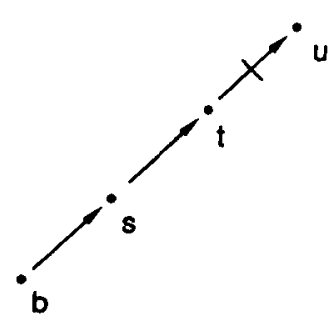

Fig. 2. $\Gamma_{2}$. 
ted paths through the process of backward chaining, according to which, at the inductive step, a compound permitted path of the form $x \rightarrow y \rightarrow \sigma$ is assembled by adding the direct link $x \rightarrow y$ to its permitted end segment $y \rightarrow \sigma$.

The present treatment, on the other hand, is intended to capture a kind of bottom-up approach to inheritance reasoning. This approach seems especially natural when one wants to push the analogy, as we do, between paths and arguments-since arguments, at least as they are usually represented (say, by proof sequences), tend to move from the beginning forward. Formally, the bottom-up approach leads to the construction of compound paths through the process of forward chaining: at the inductive step, the compound permitted path $\sigma \rightarrow x \rightarrow y$ is assembled by adding the direct link $x \rightarrow y$ to its permitted initial segment $\sigma \rightarrow x$; and likewise, the compound permitted path $\sigma \rightarrow x \rightarrow y$ is assembled by adding the direct link $x \rightarrow y$ to the permitted initial segment $\sigma \rightarrow x$. This adherence to forward chaining is one of the central principles guiding our approach. Not only does it embody a different metaphor for inheritance reasoning ("argument construction" instead of "property flow"), but it leads also to different technical results, as illustrated by our discussion of the net $\Gamma_{15}$ in Section 5.5, below. ${ }^{3}$

\subsection{Restricted skepticism}

In our approach, then, compound permitted paths are constructed through forward chaining, but of course, not every path constructible through forward chaining from the materials in a given net should be permitted by that net. Conflicts can interfere, as in the net $\Gamma_{3}$ (Fig. 3). This net has come to be known as the Nixon Diamond, because of the interpretation, due to Reiter, under which $a=$ Nixon, $q=$ Quakers, $r=$ Republicans, and $p=$ Pacifists. What $\Gamma_{3}$

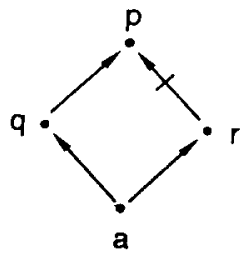

Fig. 3. $\Gamma_{3}$.

\footnotetext{
'Of course, the very description of the kind of network-based reasoning we study here as "inheritance" reasoning itself seems to suggest the top-down or "property flow" pictureaccording to which individuals are supposed to "inherit" properties from their superiors in a network roughly as one might inherit, say, the family jewels from Aunt Martha. Once one adopts the bottom-up approach, the terminology of "inheritance" is no longer so appropriate; but the terminology by now has become fixed, and it would introduce more confusion than it would eliminate if we tried to characterize this kind of reasoning process in a phrase more nearly neutral between the top-down and the bottom-up views.
} 
tells us explicitly, under this interpretation, is that Nixon is both a Quaker and a Republican, that Quakers are pacifists, and that Republicans are not pacifists. Unrestricted forward chaining would allow us to construct from this information both the paths $a \rightarrow q \rightarrow p$ and $a \rightarrow r \rightarrow p$. But since these two paths conflict, enabling the conflicting statements $a \rightarrow p$ and $a \nrightarrow p$, we don't want $\Gamma_{3}$ to permit both these paths at once. Given just the information contained in $\Gamma_{3}$, we wouldn't want to conclude both that Nixon is a pacifist and that he isn't.

What you say about inheritance depends crucially on your treatment of nets like the Nixon Diamond, which contain compound conflicting paths. One option is to suppose, although you can't permit both of two such paths, that it is reasonable to permit one or the other. In the case of the Nixon Diamond, for example, this strategy would lead us to the conclusion that either the path $a \rightarrow q \rightarrow p$ or the path $a \rightarrow r \nrightarrow p$ should be permitted. What lies behind this strategy is a kind of credulity or belief-hunger - the idea that it's best to draw as many conclusions as possible from a given net, even at the cost of making arbitrary choices among conflicting arguments. As developed by Touretzky [22], this strategy involves associating with each net containing compound conflicting paths a number of consistent extensions, which he refers to as "grounded expansions," reminiscent of the "extensions" of Reiter [18], the "fixed points" of McDermott and Doyle [13], or the "stable expansions" of Moore [14]. For this reason, because they can consistently be associated with a number of different extensions, Touretzky describes nets like these as "ambiguous." 4

We take a different point of view. Rather than supposing that an inheritance reasoner should try to conclude as much as possible from a given net, we adopt a broadly skeptical attitude, according to which conflicting arguments tend to neutralize each other. Our basic idea, which will be explained in more detail momentarily, is that a compound path is to be neutralized by any conflicting path which is not itself preempted. Even before further explanation, however, some of the consequences of this basic skeptical intuition should be clear. Given just the information in the Nixon Diamond, for example, an inheritance reasoner guided by our skeptical reasoning strategy, won't be able to conclude either that Nixon is a pacifist or that he isn't. It won't conclude that he is a

\footnotetext{
${ }^{4}$ Some implementations of credulous reasoning avoid generating multiple extensions, in one of two ways. An interactive knowledge base maintenance system developed by Rector [17] simply disallows networks with more than one extension. It issues an error message whenever an ambiguity is introduced; the user is required to resolve the problem before proceeding further. Another approach, followed by Nado and Fikes [15] in the OPUS system, is to adopt a restricted (but still nonmonotonic) language which is incapable of expressing the kinds of assertions that give rise to multiple extensions. In the terminology of $[22,23]$, OPUS is a unipolar class/property system with monotonic, explicit exception links. It is unipolar because it does not allow negative statements. A system must either provide for negative statements or have slots restricted to holding single values (which are an implicit form of negation) in order to have multiple extensions.
} 
pacifist, since the information contained in the net provides the materials for constructing an argument to the contrary; it won't conclude that he isn't a pacifist, since the net also provides the materials for constructing an argument that he is.

Although our approach is based, generally, on the skeptical idea that such paths tend to neutralize each other, the special brand of skepticism we adopt here is restricted in two ways. First, we suppose that only compound paths can be neutralized at all; and second, that paths can be neutralized only by conflicting paths which are not themselves preempted. Both of these restrictions are important; we examine them in turn.

\subsubsection{Compound versus direct conflicts}

As an example of a net containing conflicting direct paths, consider $\Gamma_{4}$ (Fig. 4). (Again, take $a=$ Nixon and $p=$ Pacifists.) According to the definition we provide, $\Gamma_{4}$ will permit $b o t h$ the conflicting paths $a \rightarrow p$ and $a \rightarrow p$ : our reasoner will conclude from $\Gamma_{4}$ both that Nixon is a pacifist and that he isn't. This may seem odd, especially in light of our cautious, skeptical approach to $\Gamma_{3}$. It may appear, from a certain point of view, that $\Gamma_{4}$ presents us with nothing but a limiting case of the phenomenon found in $\Gamma_{3}$-so that consistency of principle should lead us to conclude, if $\Gamma_{3}$ doesn't permit either the path $a \rightarrow q \rightarrow p$ or the path $a \rightarrow r \rightarrow p$, that $\Gamma_{4}$, likewise, shouldn't permit either of the paths $a \rightarrow p$ or $a \nrightarrow p$. But it is also possible to isolate a point of view from which our different treatment of the conflicting paths in $\Gamma_{3}$ and $\Gamma_{4}$ seems just right.

Remember, we are talking about the design of an inheritance reasoner, a mechanism for drawing conclusions from a certain kind of database-a set of statements that can be represented as the set of links in a net. Now when we think of the net $\Gamma_{3}$ as a database, it is, of course, consistent: in fact, under the Nixon interpretation, all of the statements contained in $\Gamma_{3}$ are true. Obviously, no one would want a reasoning mechanism to draw inconsistent conclusions from consistent information; so it follows at once tht $\Gamma_{3}$ cannot permit both the paths $a \rightarrow q \rightarrow p$ and $a \rightarrow r \rightarrow p$, since these two paths enable the conflicting statements that Nixon is a pacifist $(a \rightarrow p)$ and that he isn't $(a \rightarrow p)$. On the other hand, when we look at $\Gamma_{4}$ as a database, it already contains both of these

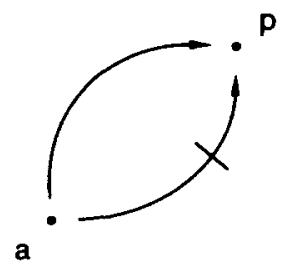

Fig. 4. $\Gamma_{4}$. 
statements; so in this case, we are faced with the problem of drawing the appropriate conclusions from information that is already inconsistent.

This is a notoriously difficult problem, but we find that it is both possible and useful to adopt in the context of inheritance reasoning a proposal that was originally formulated by Belnap, in $[1,2]$, as a guide for deductive reasoning in the presence of inconsistency. As a general principle, we propose that a reasoner ought to be able to conclude from a set of statements every statement actually contained in that set, at least-even if the set is inconsistent. It follows, of course, that if our inheritance reasoner were actually provided with the information contained in $\Gamma_{4}$-that Nixon both is and isn't a pacifist-it ought to be able to conclude from this information both that Nixon is a pacifist and that he isn't. Thinking of deductive reasoning, Belnap argues that the presence of inconsistent information shouldn't enable a mechanical reasoner to derive arbitrary conclusions, as it would in the case of a theorem prover using classical logic. We have shown in [21], however, that this much of the motivation behind relevance logic is already built into inheritance reasoning, even in the simple case of monotonic inheritance; and the arguments we provided there carry over into the nonmonotonic case. Thus, the reasoner we describe in this paper will conclude from $\Gamma_{4}$, as it should, both that Nixon is a pacifist and that he isn't; but it won't then go to draw irrelevant conclusions from this conflict: it won't conclude, for instance, that Nixon is a Democrat.

\subsubsection{Preemption}

The second restriction on our broadly skeptical outlook is the idea that even compound arguments are neutralized only by those conflicting arguments that are not themselves preempted. This idea-that certain compound arguments can be, as we say, preempted by others-really lies at the heart of our approach, allowing us to transform a simple and dogmatic skepticism into something much more interesting.

Again, we begin with an example, the net $\Gamma_{5}$ (Fig. 5). This net results from adding the link $p \leftrightarrow r$ to $\Gamma_{1}$, and the interpretations of these two nets will overlap as well. Just as before, we take $a=$ Tweety, $q=$ Birds, and $r=$ Flying Things; but now let's shift the earlier interpretation so that $p=$ Penguins,

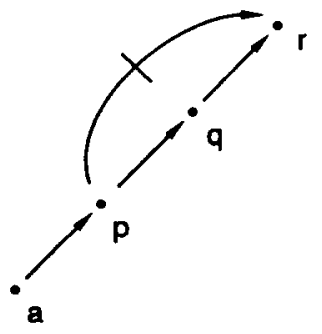

Fig. 5. $\Gamma_{5}$. 
giving some plausibility to the new link $p \nrightarrow r$. If things are like this, what are we to conclude about Tweety: does he fly or not? Well, there are two paths to consider: $a \rightarrow p \rightarrow q \rightarrow r$, which enables the conclusion that Tweety flies, and $a \rightarrow p \nrightarrow r$, which enables the opposite conclusion. Since both of these paths are compound, and they enable conflicting conclusions, simple skepticism would bar us from reaching any conclusion at all. But evidently, in this case, we do want to reach a conclusion: we want to conclude, in fact, that Tweety doesn't fly-since he is a penguin, and penguins don't fly. The reason we are able to conclude here that Tweety doesn't fly-even though he is a bird, and birds fly-is that penguins happen to be a specific kind of bird, so that, in case of conflicts, the information we have about Tweety in virtue of his being a penguin overrides whatever we would otherwise suppose to be true of him simply because he is a bird.

This illustrates the central intuition behind preemption: that arguments based on more specific information override arguments based on less specific information. As we define it, a path will be preempted in a net, roughly, when the net provides the materials for constructing a conflicting argument based on more specific information. Looking again at the net $\Gamma_{5}$, we see that it both permits the path $a \rightarrow p \rightarrow q$ (telling us that Tweety is a bird) and contains the link $q \rightarrow r$ (telling us directly that birds fly). We want to say, however, that the path $a \rightarrow p \rightarrow q \rightarrow r$ (telling us that Tweety flies, because he is a bird and birds fly) is preempted in $\Gamma_{5}$, since the net also contains the link $p \nrightarrow q$ (telling us directly that penguins don't fly), and conclusions deriving from the node $p$ (penguins) are based on more specific information about $a$ (Tweety) than conclusions deriving from the node $q$ (birds). In terms of the topology of $\Gamma_{5}$, it's natural to suppose that the reason $p$ can be said to provide more specific information about $a$ than $q$ does is simply that the net permits the $a$ path from a through $p$ to $q$; this path, $a \rightarrow p \rightarrow q$, tells us both that Tweety is a penguin and that penguins are a specific kind of bird. So, restating in a way that incorporates this analysis of specificity, we can say that the path $a \rightarrow p \rightarrow q \rightarrow r$ is preempted in $\Gamma_{5}$ just because there is a node $p$ such that $\Gamma_{5}$ both contains the direct link $p \rightarrow r$ and permits the path $a \rightarrow p \rightarrow q$.

This idea of preemption can easily be generalized to apply to arbitrary nets and paths. We will say that a path of the form $x \rightarrow \tau \rightarrow v \rightarrow y$ is preempted in a net $\Gamma$ just in case there is a node $z$ such that $z \nrightarrow y \in \Gamma$, and either $z=x$ or $\Gamma$ permits a path of the form $x \rightarrow \tau_{1} \rightarrow z \rightarrow \tau_{2} \rightarrow v$. With exact symmetry, we say that a path of the form $x \rightarrow \tau \rightarrow v \rightarrow y$ is preempted in a net $\Gamma$ just in case there is a node $z$ such that $z \rightarrow y \in \Gamma$ and either $z=x$ or $\Gamma$ permits a path of the form $x \rightarrow \tau_{1} \rightarrow z \rightarrow \tau_{2} \rightarrow v$.

\section{Defining Inheritance}

Let's use the symbol " $p$ " to stand for the permission relation, so that " $\Gamma p \sigma$ " means that the net $\Gamma$ permits the path $\sigma$. We have now considered the central 
principles underlying our approach to this idea-forward chaining, along with a certain kind of restricted skepticism. It remains only to organize these principles into a rigorous definition.

\subsection{Degree}

Our adoption of forward chaining suggests that a bottom-up, inductive definition should be possible. In order to frame such a definition, however, we need to be able to associate with each path $\sigma$ some measure of its "complexity" in a given net $\Gamma$, in such a way that it can be decided whether $\Gamma \gg \sigma$ once it is known whether $\Gamma P \sigma^{\prime}$ for each path $\sigma^{\prime}$ less complex in $\Gamma$ than $\sigma$ itself.

The natural thing to think is that we might be able to identify the complexity of a path, in this sense, with its length - but this won't work, since we will often need to know about longer paths before we can decide whether shorter paths are permitted. As an example, consider the net $\Gamma_{6}$ (Fig. 6). Here, it follows at once from our motivating principles that the path $x \rightarrow p \rightarrow y$, which has length two, shouldn't be permitted, since it is compound and it conflicts with the unpreempted path $x \rightarrow q \rightarrow r \rightarrow y$, of length three. A more complicated situation arises in the case of $\Gamma_{7}$ (Fig. 7). Here, the path $x \rightarrow p \rightarrow y$ should be permitted: the potentially conflicting path $x \rightarrow q \rightarrow r+y$ no longer interferes, since it is itself neutralized by the path $x \rightarrow s \rightarrow t \rightarrow u \rightarrow r$, which is longer still. Evidently, as this net suggests, before we can decide whether a particular path is to be permitted, we need to know abcut all the conflicting paths that might neutralize it, as well as all the paths that might neutralize those conflicting paths, all the other paths that might neutralize those, and so on.

There are several ways to order the paths in a given net so that an inductive definition, as it steps through the ordering, will wind up considering all the paths relevant to a particular path before it considers that path itself. We adopt here what seems to be the simplest such ordering; later, in Section 6.2.1, we describe a slightly more complicated ordering, with other compensating virtues. To get at our simple ordering, we first introduce an auxiliary idea. As we recall from Section 2.1, a path is a joined sequence of links containing a negative link, if at all, only at the very end. Let's say, now, that a generalized path is a sequence of links joined like an ordinary path, except that it can

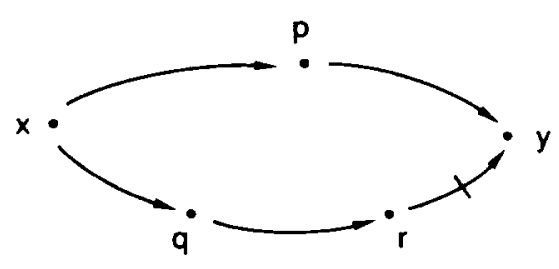

Fig. 6. $\Gamma_{6}$.

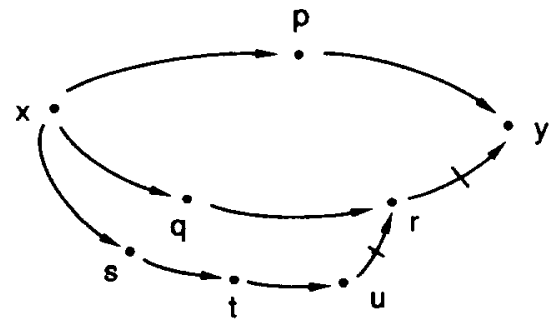

Fig. $7 . \Gamma_{7}$ 
contain negative links anywhere, and perhaps more than one. Formally, we can catch this idea by specifying that each assertion is a generalized path, and that, if $\sigma$ is a generalized path, then both $\sigma \rightarrow y$ and $\sigma \rightarrow y$ are generalized paths. (It follows, of course, that the generalized paths include the ordinary paths.) Using this auxiliary concept of a generalized path, we now define the degree of a path $\sigma$ in $a$ net $\Gamma$-written, $\operatorname{deg}_{I}(\sigma)$-as the length of the longest generalized path in $\Gamma$ from the initial node of $\sigma$ to its end node. Unlike length, degree is not a property of paths alone, but of paths in a net. For example, we have

$$
\begin{aligned}
& \operatorname{deg}_{I_{6}}(x \rightarrow p \rightarrow y)=3, \\
& \operatorname{deg}_{I_{7}}(x \rightarrow p \rightarrow y)=5,
\end{aligned}
$$

since in $\Gamma_{6}$, the longest generalized path from $x$ to $y$ (which happens to be an ordinary path) has length three, while $\Gamma_{7}$ contains a (true) generalized path from $x$ to $y$ of length five.

As it turns out, this idea of degree provides an acceptable notion of path "complexity" for an inductive definition of $p$, the permission relation between nets and paths: it can be decided whether $\Gamma p \sigma$ entirely on the basis of information regarding paths whose degree in $\Gamma$ is less than that of $\sigma$, along with information about the direct links contained in $\Gamma$ itself. On the other hand, in order to assure that $\operatorname{deg}_{\Gamma}(\sigma)$ should always be well-defined, we need to restrict our attention to nets which are acyclic, in the sense that they contain no generalized paths whose initial nodes are identical with their end nodes. (This is a common restriction; much of the analysis in Touretzky [22], for instance, also applies only to acyclic nets.)

\subsection{The definition}

Given this idea of degree, then, and restricting ourselves to acyclic nets, we can now present our definition of the permission relation. Although the definition is inductive at heart, it has the overall structure of a definition by cases: it deals separately with compound paths and with direct links (non-compound paths). Only in the case of compound paths is there any need to resort to induction; direct links can be handled all at once, as follows.

Case I: $\sigma$ is a direct link. Then $\Gamma \gg \sigma$ iff $\sigma \in \Gamma$.

It is important to note that even if $\sigma$ is a direct link, it could easily turn out that $\operatorname{deg}_{\Gamma}(\sigma)>1$, since $\Gamma$ might contain a compound generalized path from the initial node of $\sigma$ to its end node. On the other hand, if $\operatorname{deg}_{\Gamma}(\sigma)=1$, then the path $\sigma$ has to be a direct link. Thus, in addition to taking care of all the direct links at once, whatever their degree, Case I serves also as the basis clause for the induction on degree which extends the permission relation from direct links to compound paths. The inductive clause is as follows.

Case II: $\sigma$ is a compound path with, say, $\operatorname{deg}_{\Gamma}(\sigma)=n$. As an inductive 
hypothesis, we can suppose it is settled whether $\Gamma \triangleright \sigma^{\prime}$ whenever $\operatorname{deg}_{\Gamma}\left(\sigma^{\prime}\right)<n$. There are then two subcases to consider, depending on the form of $\sigma$.

(1) $\sigma$ is a positive path, of the form $x \rightarrow \sigma_{1} \rightarrow u \rightarrow y$. Then $\Gamma \ngtr \sigma$ iff

(a) $\Gamma p x \rightarrow \sigma_{1} \rightarrow u$,

(b) $u \rightarrow y \in \Gamma$,

(c) $x+y \not \subset \Gamma$,

(d) for all $v$ and $\tau$ such that $\Gamma \triangleright x \rightarrow \tau \rightarrow v$ with $v \nrightarrow y \in \Gamma$, there exist $z, \tau_{1}$ and $\tau_{2}$ such that $z \rightarrow y \in \Gamma$ and either $z=x$ or $\Gamma \triangleright x \rightarrow \tau_{1} \rightarrow z \rightarrow \tau_{2} \rightarrow v$.

(2) $\sigma$ is a negative path, of the form $x \rightarrow \sigma_{1} \rightarrow u \rightarrow y$. Then $\Gamma \ngtr \sigma$ iff

(a) $\Gamma \gg x \rightarrow \sigma_{1} \rightarrow u$,

(b) $u \rightarrow y \in \Gamma$,

(c) $x \rightarrow y \notin \Gamma$

(d) for all $v$ and $\tau$ such that $\Gamma \gg x \rightarrow \tau \rightarrow v$ with $v \rightarrow y \in \Gamma$, there exist $z, \tau_{1}$ and $\tau_{2}$ such that $z \nrightarrow y \in \Gamma$ and either $z=x$ or $\Gamma \triangleright x \rightarrow \tau_{1} \rightarrow z \rightarrow \tau_{2} \rightarrow v$.

It should be clear that this definition of the permission relation accurately represents the general approach to inheritance reasoning described in Section 3. Case I tells us that any statement actually contained in a net should be permitted by that net. The two subcases of Case II, dealing respectively with positive and negative compound paths, are perfectly symmetric. In each subcase, the clauses (a) and (b) capture the idea of forward chaining: compound paths are permitted by a net only if they can be constructed by adding direct links from the net to initial permitted segments of those paths. The clauses (c) and (d) take care of conflicts. What (d) says is that, even if a compound path is constructible through forward chaining, it can be permitted only if each potentially conflicting compound path is preempted. Of course, only compound conflicting paths can actually be preempted, since preemption involves the intermediate nodes of a path, and direct links have no intermediate nodes; but if, for skeptical reasons, we don't want a path to be permitted which conflicts with an unpreempted compound path, we certainly don't want to permit a path that conflicts with a direct link. This is the force of the clause (c).

Both the clauses (a) and (d) in the inductive step refer to other paths of a certain form permitted by the net; but this is no problem, because at any step in the induction, paths of this form will always have a degree less than that of the path being considered.

\section{Discussion}

It is easy to see that the definition of the permission relation presented in Section 4 yields the advertised results applied to the nets $\Gamma_{1}$ through $\Gamma_{5}$ from 
Section 3. In this section we explore certain properties of the definition. We show that the treatment of inheritance embodied in this definition is nonmonotonic, that it is sound, and that it has the property of atomic stability. We show that the skeptical theory of a network is not identical with the intersection of its credulous theories, and that it allows for decoupling of conclusions.

\subsection{Nonmonotonicity}

The analysis presented here is put forth as an analysis of nonmonotonic inheritance: paths permitted by a net may no longer be permitted once that net is supplemented with additional links; statements supported by a net may no longer be supported once that net is supplemented with additional links.

The simplest kind of counterexample to monotonicity is illustrated by the nets $\Gamma_{8}$ and $\Gamma_{9}$ (Figs. 8 and 9). Here, $\Gamma_{8}$ permits the path $a \rightarrow q \rightarrow p$, and so supports the statement $a \rightarrow p$. On the other hand, $\Gamma_{9}$ neither permits the path nor supports the statement; but of course $\Gamma_{8} \subseteq \Gamma_{9}$. From the standpoint of our general skeptical motivation, this is as it should be. Imagine that the nodes in these two nets are interpreted as in the Nixon Diamond, with $a=$ Nixon, $q=$ Quakers, and $p=$ Pacifists. Then $\Gamma_{8}$ gives us the materials for constructing an argument, represented by the path $a \rightarrow q \rightarrow p$, for the conclusion that Nixon is a pacifist, but in the net $\Gamma_{9}$, both this argument and its conclusion are neutralized by the direct statement that Nixon is not a pacifist.

A different kind of counterexample to monotonicity-relying on preemption rather than a conflict with direct links-is provided by the nets $\Gamma_{1}$ and $\Gamma_{5}$ from Section 3. Again, $\Gamma_{1}$ is a subset of $\Gamma_{5}$. However, $\Gamma_{1}$ permits the path $a \rightarrow p \rightarrow q \rightarrow r$, and so supports the statement $a \rightarrow r ; \Gamma_{5}$ neither permits the path nor supports the statement.

\subsection{Soundness}

A reasoning mechanism should be sound, at least in the sense that it never leads from a consistent set of assumptions to an inconsistent conclusion. Classical deductive systems, for example, are sound in this sense; but of course, if a reasoner based on classical logic were ever supplied with an inconsistent set of assumptions, it would then support any conclusion at all.

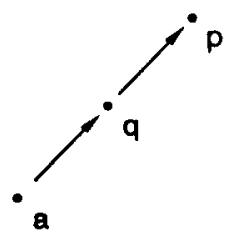

Fig. $8 . \Gamma_{8}$

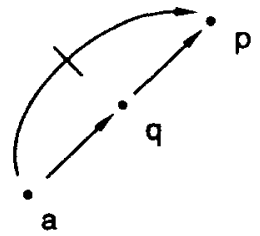

Fig. 9. $\Gamma_{9}$. 
Like classical logic, the inheritance reasoner we describe here is also sound in this standard sense. However, as we mentioned earlier, in Section 3.2.1, it is designed to behave more sensibly than classical logic in the presence of conflicting information. Although this reasoner will conclude from a network containing conflicting statements every statement actually contained in that network, the effect of these conflicts is localized: they don't lead the reasoner to other, possibly irrelevant conclusions. The following theorem shows that the reasoner we describe won't ever draw conflicting conclusions from a network unless that network already contains conflicting statements, as conflicting direct links; and even if the network does happen to contain conflicting statements, our reasoner won't draw any conflicting conclusions that aren't already contained in the network-it will never draw any new conflicting conclusions.

Theorem 5.1. If $\Gamma$ supports both $x \rightarrow y$ and $x \rightarrow y$, then both $x \rightarrow y \in \Gamma$ and $x \nrightarrow y \in \Gamma$.

Proof. Suppose $\Gamma$ supports both $x \rightarrow y$ and $x \rightarrow y$, but doesn't contain both $x \rightarrow y$ and $x+y$. Then either (i) $\Gamma$ supports exactly one of these statements only through compound paths, or (ii) $\Gamma$ supports both of these statements only through compound paths. It is easy to see from clauses II.1(c) and II.2(c) of the inheritance definition that (i) is impossible: if $\Gamma$ contains either statement, it can't permit a compound path enabling the other. We prove the theorem by showing that (ii) is impossible as well.

Suppose (ii) is true. Then $\Gamma$ permits some path of the form $x \rightarrow \sigma_{1} \rightarrow u_{1} \rightarrow y$, and also path of the form $x \rightarrow \sigma_{2} \rightarrow u_{2} \nrightarrow y$. Since $\Gamma$ permits a compound path from $x$ to $y$, it follows from II.1(a)-(b) and II.2(a)-(b) that $\Gamma$ permits some path of the form $x \rightarrow \tau \rightarrow v$ with either $v \rightarrow y \in \Gamma$ or $v \rightarrow y \in \Gamma$. Let $x \rightarrow \tau^{\prime} \rightarrow v^{\prime}$ be a path of minimal degree satisfying this condition-that is, $\Gamma$ permits $x \rightarrow \tau^{\prime} \rightarrow v^{\prime}$ and either $v^{\prime} \rightarrow y \in \Gamma$ or $v^{\prime} \nrightarrow y \in \Gamma$; and there is no path $x \rightarrow \tau^{\prime \prime} \rightarrow v^{\prime \prime}$ with

$$
\operatorname{deg}_{\Gamma}\left(x \rightarrow \tau^{\prime \prime} \rightarrow v^{\prime \prime}\right)<\operatorname{deg}_{\Gamma}\left(x \rightarrow \tau^{\prime} \rightarrow v^{\prime}\right)
$$

such that $\Gamma$ permits $x \rightarrow \tau^{\prime \prime} \rightarrow v^{\prime \prime}$ and either $v^{\prime \prime} \rightarrow y \in \Gamma$ or $v^{\prime \prime} \nrightarrow y \in \Gamma$. Suppose $v^{\prime} \rightarrow y \in \Gamma$. From the assumption that (ii), it follows that there are no direct links in $\Gamma$ from $x$ to $y$. Therefore, since $\Gamma$ permits $x \rightarrow \sigma_{2} \rightarrow u_{2} \nrightarrow y$, it follows from II.2(d) that there must be a node $z$ such that $\Gamma \triangleright x \rightarrow \tau_{1} \rightarrow z \rightarrow \tau_{2} \rightarrow v^{\prime}$ and $z \nrightarrow y \in \Gamma$. From this it follows that $\Gamma$ permits $x \rightarrow \tau_{2} \rightarrow z$, where

$$
\operatorname{deg}_{\Gamma}\left(x \rightarrow \tau_{2} \rightarrow z\right)<\operatorname{deg}_{\Gamma}\left(x \rightarrow \tau^{\prime} \rightarrow v^{\prime}\right)
$$

and $z \rightarrow y \in \Gamma$ or $z \nrightarrow y \in \Gamma$. Therefore, the path $x \rightarrow \tau^{\prime} \rightarrow v^{\prime}$ cannot be of minimal degree, contrary to assumption. If $v^{\prime} \rightarrow y \in \Gamma$, it follows likewise from 
the assumption that $\Gamma$ permits $x \rightarrow \sigma_{1} \rightarrow u_{1} \rightarrow y$ and II.1(d) that $x \rightarrow \tau^{\prime} \rightarrow v^{\prime}$ cannot be of minimal degree.

\subsection{Stability}

The property of stability is the property of being insensitive in certain ways to redundant information. In general, there are a number of different stability properties we might choose to require in an acceptable reasoner, and the differences between them can be subtle. Just to illustrate the kind of thing that goes wrong when an inheritance reasoner is entirely unstable, however, let's consider for a moment a shortest-path reasoning algorithm, such as that suggested by Fahlman [5], which resolves differences among conflicting paths by favoring the shortest.

Consider the nets $\Gamma_{10}$ and $\Gamma_{11}$ (Figs. 10 and 11 ). Evidently, $\Gamma_{11}$ results from $\Gamma_{10}$ simply through the addition of the link $a \rightarrow r$, which is both atomic and redundant - in the sense that shortest-path reasoning applied to $\Gamma_{10}$ already tells us that the statement represented by this link is true. Still, even though this atomic link is redundant from the standpoint of $\Gamma_{10}$, adding it to $\Gamma_{10}$ changes the semantics of that net, according to the shortest-path approach to inheritance reasoning: while $\Gamma_{10}$ supports the statement $a \rightarrow s$ (since the path $a \rightarrow p \rightarrow q \rightarrow s$ is shorter than the conflicting path $a \rightarrow p \rightarrow q \rightarrow r \rightarrow s$ ), the new net $\Gamma_{11}$ would support the statement $a \rightarrow s$ instead (since the path $a \rightarrow r \rightarrow s$ is now shorter than the conflicting path $a \rightarrow p \rightarrow q \rightarrow s$ ).

The example works out differently according to the analysis of inheritance presented here (as well as that of [22]). On the present analysis, it is clear that the two nets $\Gamma_{10}$ and $\Gamma_{11}$ support exactly the same statements. In particular, $\Gamma_{11}$ doesn't now permit the path $a \rightarrow r \rightarrow s$; so, like $\Gamma_{10}$, the net $\Gamma_{11}$ doesn't support the statement $a \rightarrow s$. This situation illustrates the following atomic stability theorem, which shows that the reasoner we describe is stable with respect to redundant atomic statements in a way that shortest-path reasoning is not.

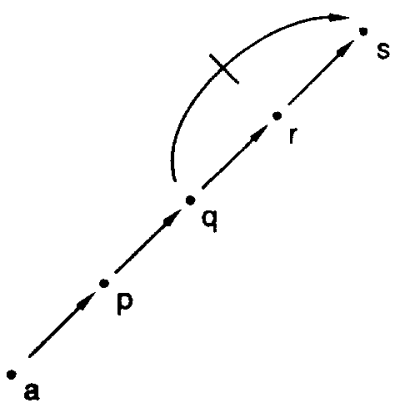

Fig. 10. $\Gamma_{10}$.

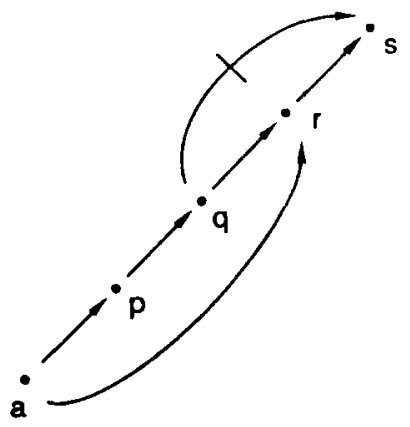

Fig. 11. $\Gamma_{11}$. 
Theorem 5.2. For an atomic statement $A$, if $\Gamma$ supports $A$, then for any statement $B, \Gamma \cup\{A\}$ supports $B$ if and only if $\Gamma$ supports $B$.

The theorem is an immediate consequence of the following more general lemma, which establishes a connection between the set of paths permitted by a net, and the set of paths permitted once that net is supplemented with a redundant atomic statement.

Lemma 5.3. (a) Suppose $\Gamma \ngtr a \rightarrow \delta \rightarrow p$. It follows that, if $\Gamma \ngtr \sigma$, then $\Gamma \cup$ $\{a \rightarrow p\} P \sigma$; and also that, if $\Gamma \cup\{a \rightarrow p\} \vee \sigma$, then $\Gamma \triangleright \sigma^{*}$, where $\sigma^{*}$ is the result of replacing any occurrence of the link $a \rightarrow p$ in $\sigma$ by the path $a \rightarrow \delta \rightarrow p$. (b) Likewise, suppose $\Gamma \gg a \rightarrow \delta \rightarrow p$. It then follows that, if $\Gamma \gg \sigma$, then $\Gamma \cup\{a \rightarrow p\} \ngtr \sigma$, and also, if $\Gamma \cup\{a \rightarrow p\} \ngtr \sigma$, that $\Gamma \gg \sigma^{*}$, where $\sigma^{*}$, now, is the result of replacing any occurrence of the link $a \nrightarrow p$ in $\sigma$ by the path $a \rightarrow \delta \nrightarrow p$.

Proof. We prove only (a); the proof of (b) is similar. First, suppose $\sigma$ is a direct link. Let $\Gamma \triangleright \sigma$. Then we know from Case I of the definition that $\sigma \in \Gamma$; so $\sigma \in \Gamma \cup\{a \rightarrow p\}$; so $\Gamma \cup\{a \rightarrow p\} \ngtr \sigma$. Now let $\Gamma \cup\{a \rightarrow p\} \ngtr \sigma$. If $\sigma \neq$ $a \rightarrow p$, then $\sigma \in \Gamma$, and also $\sigma=\sigma^{*}$; so $\Gamma \ngtr \sigma^{*}$. If $\sigma=a \rightarrow p$, then $\sigma^{*}=$ $a \rightarrow \delta \rightarrow p$; so $\Gamma P \sigma^{*}$ by assumption.

Next, suppose $\sigma$ is a compound path, with $\operatorname{deg}_{\Gamma \cup\{a \rightarrow p\}}(\sigma)=n$. As an inductive hypothesis, we suppose that for all $\sigma^{\prime}$ with $\operatorname{deg}_{\Gamma \cup\{a \rightarrow p\}}\left(\sigma^{\prime}\right)<n$, we know both (i) that $\Gamma \cup\{a \rightarrow p\} P \sigma^{\prime}$ if $\Gamma \triangleright \sigma^{\prime}$ and (ii) that $\Gamma \ngtr \sigma^{\prime *}$ if $\Gamma \cup\{a \rightarrow p\} \ngtr \sigma^{\prime}$. To carry out the inductive step of the proof, we need to consider two subcases, depending on whether $\sigma$ is a positive or a negative compound path; and for each subcase, it is necessary to show both that $\Gamma \cup\{a \rightarrow p\} \ngtr \sigma$ if $\Gamma \ngtr \sigma$, and that $\Gamma \triangleright \sigma^{*}$ if $\Gamma \cup\{a \rightarrow p\} \ngtr \sigma$. The cases are largely similar; we show here ony that if $\sigma$ is a positive compound path, of the form $x \rightarrow \sigma_{1} \rightarrow u \rightarrow y$, then $\Gamma \ngtr \sigma^{*}$ whenever $\Gamma \cup\{a \rightarrow p\} \ngtr \sigma$. that

Suppose that $\Gamma \cup\{a \rightarrow p\} P \sigma$. We know from Case II.1 of the definition

(a) $\Gamma \cup\{a \rightarrow p\} \ngtr x \rightarrow \sigma_{1} \rightarrow u$,

(b) $u \rightarrow y \in \Gamma \cup\{a \rightarrow p\}$,

(c) $x \rightarrow y \notin \Gamma \cup\{a \rightarrow p\}$,

(d) for all $v$ such that $\Gamma \cup\{a \rightarrow p\} \ngtr x \rightarrow \tau \rightarrow v$ with $v \rightarrow y \in \Gamma \cup\{a \rightarrow p\}$, there exists $z$ such that $z \rightarrow y \in \Gamma \cup\{a \rightarrow p\}$ and either $z=x$ or $\Gamma \cup$ $\{a \rightarrow p\} P x \rightarrow \tau_{1} \rightarrow z \rightarrow \tau_{2} \rightarrow v$.

From (a) and (ii) of the inductive hypothesis, we can conclude that

$\left(\mathrm{a}^{*}\right) \Gamma \triangleright\left(x \rightarrow \sigma_{1} \rightarrow u\right)^{*}$.

We know that $u \rightarrow y \neq a \rightarrow p$, since $a$ is an individual and $u$ must be a kind; so 
we can conclude from (b) that

(b*) $u \rightarrow y \in \Gamma$.

It follows directly from (c) that

(c*) $x \rightarrow y \notin \Gamma$.

Finally, suppose that, for some $v, \Gamma \gg x \rightarrow \tau \rightarrow v$ with $v \nrightarrow y \in \Gamma$. Of course, $v \rightarrow y \in \Gamma \cup\{a \rightarrow p\}$ as well, and we know from (i) of the inductive hypothesis that $\Gamma \cup\{a \rightarrow p\} P x \rightarrow \tau \rightarrow v$. Therefore (d) above tells us that there exists $z$ such that $z \rightarrow y \in \Gamma \cup\{a \rightarrow p\}$ and either $z=x$ or $\Gamma \cup$ $\{a \rightarrow p\} P x \rightarrow \tau_{1} \rightarrow z \rightarrow \tau_{2} \rightarrow v$. Here, there are two cases to consider: either $z \rightarrow y \neq a \rightarrow p$ or $z \rightarrow y=a \rightarrow p$. In the first case, we have $z \rightarrow y \in \Gamma$. If $\Gamma \cup\{a \rightarrow p\} P x \rightarrow \tau_{1} \rightarrow z \rightarrow \tau_{2} \rightarrow v$, then we can conclude from (ii) of the inductive hypothesis that $\Gamma P\left(x \rightarrow \tau_{1} \rightarrow z \rightarrow \tau_{2} \rightarrow v\right)^{*}$. Thus we know, in this case, that there exists $z$ such that $z \rightarrow y \in \Gamma$ and either $z=x$ or $\Gamma P\left(x \rightarrow \tau_{1} \rightarrow z \rightarrow \tau_{2} \rightarrow v\right)^{*}$. In the second case, we must have $z=x=a$, since $a$ is an individual, and also $y=p$, so that $x \rightarrow \rightarrow \tau v \rightarrow y=a \rightarrow \tau \rightarrow v \rightarrow p$. But by hypothesis, we have $\Gamma P a \rightarrow \delta \rightarrow p$, and also by hypothesis $\Gamma \triangleright x \rightarrow \tau \rightarrow v$ and $v \rightarrow y \in \Gamma$-that is, $\Gamma P a \rightarrow \tau \rightarrow v$ and $v \rightarrow p \in \Gamma$. Hence the path $a \rightarrow \tau \rightarrow v \nrightarrow p$ must be preempted in $\Gamma$ : there must be a $z^{\prime}$ such that $z^{\prime} \rightarrow p \in \Gamma$ and either $z^{\prime}=a$ or $\Gamma \gg z \rightarrow \tau_{1}^{\prime} \rightarrow z^{\prime} \rightarrow \tau_{2}^{\prime} v$. But then $z^{\prime} \rightarrow y \in \Gamma$ (since $y=p$ ) and either $z^{\prime}=x$ (since $x=a$ ) or $\Gamma P z \rightarrow \tau_{1}^{\prime} \rightarrow z^{\prime} \rightarrow \tau_{2}^{\prime} \rightarrow v$. Combining these two cases, we can see that

$\left(\mathrm{d}^{*}\right)$ for all $v$ such that $\Gamma \gg x \rightarrow \tau \rightarrow v$ with $v \nrightarrow y \in \Gamma$, there exists $z$ such that $z \rightarrow y \in \Gamma$ and either $z=x$ or $\Gamma P\left(x \rightarrow \tau_{1} \rightarrow z \rightarrow \tau_{2} \rightarrow v\right)^{*}$.

And from $\left(\mathrm{a}^{*}\right)$ through $\left(\mathrm{d}^{*}\right)$, we can conclude that $\Gamma \triangleright \sigma^{*}$.

What Theorem 5.2 shows, again, is that you can't affect the set of statements supported by a net by supplementing it with atomic statements it already supports. The analog to this theorem fails, however, when a net is supplemented with a redundant generic statement. To see this, consider the nets $\Gamma_{12}$ and $\Gamma_{13}$ (Figs. 12 and 13). Here, $\Gamma_{12}$ doesn't permit the path $a \rightarrow p \rightarrow q \rightarrow r \rightarrow s$ (since its initial segment $a \rightarrow p \rightarrow q \rightarrow r$ is preempted); so this net doesn't support the statement $a \rightarrow s$. On the other hand, since $\Gamma_{12}$ permits the path $q \rightarrow r \rightarrow s$, it does support the generic statement $q \rightarrow s$. Evidently, $\Gamma_{13}$ results from $\Gamma_{12}$ only through the addition of this statement, redundant from the standpoint of $\Gamma_{12}$. Yet, $\Gamma_{13}$ does now support the statement $a \rightarrow s$, since $\Gamma_{13}$ permits $a \rightarrow p \rightarrow q \rightarrow s$.

It's hard to know what to make of examples like this. We view it almost as a criterion of acceptability in an inheritance reasoner that it should exhibit atomic stability. No one has ever produced a counterexample to atomic stability with any intuitive force; and it was, in part, the failure of this property 


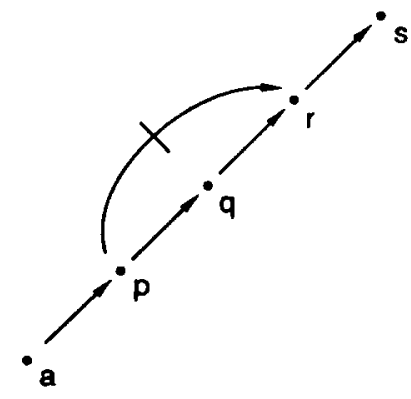

Fig. 12. $\Gamma_{12}$.

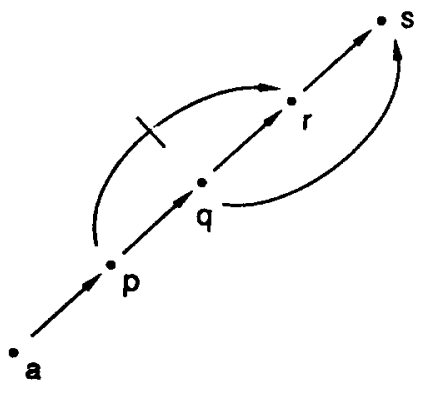

Fig. 13. $\Gamma_{13}$.

in shortest-path reasoners that motivated both the theory of Touretzky [22] and the present analysis. When it comes to generic stability, however, the matter is more complicated.

On one hand, it is surprising - at least from the standpoint of our analogy between inheritance reasoning and deductive reasoning - to find that an acceptable inheritance reasoner might fail to exhibit generic stability. The deductive analog of a network is a set of hypotheses, or axioms; the statements supported by a network are like the theorems derivable from those axioms. From the standpoint of our analogy, then, the failure of stability is like a situation in which the consequences of a set of axioms would be affected if the axioms were supplemented, not just with an arbitrary statement, but with a theorem derivable from those axioms - and this makes little sense even from a general deductive point of view, allowing for the possibility of nonmonotonic deductive systems. ${ }^{5}$ On the other hand, even though it indicates a break in our analogy between inheritance and deduction, it is possible to argue that the kind of generic instability exhibited above might actually turn out to be a desirable property in an inheritance reasoner: it suggests a way in which the graphtheoretic nature of inheritance reasoning allows a kind of sensitivity to the structure of arguments that is difficult to achieve in deductive systems.

To illustrate, we supply $\Gamma_{12}$ and $\Gamma_{13}$ with the following interpretation, inspired by an example from Sandewall [20]: $a=$ Moby, $p=$ Whales, $q=$ Mammals, $r=$ Land-dwellers, $s=$ Air-breathers. On this interpretation, what $\Gamma_{12}$ tells us directly is that Moby is a whale, that whales are mammals, that mammals are land-dwellers, that whales aren't land-dwellers, and that land-dwellers are air-breathers. Given just this information, we shouldn't be able to conclude that Moby is an air-breather: only land-dwellers are known directly to be

\footnotetext{
${ }^{5}$ In a deductive system with consequence relation $\vdash$, monotonicity is the principle that if $\Gamma \vdash A$ then $\Gamma \cup \Delta \vdash A$; stability is the principle that if $\Gamma \vdash A$ then $\Gamma \cup\{A\} \vdash B$ iff $\Gamma \vdash B$. Even nonmonotonic consequence relations - such as the relation $\vdash_{P}$ defined by McCarthy [12]-tend to be stable.
} 
air-breathers, and we can conclude that Moby isn't a land-dweller, since he is a whale. Of course, $\Gamma_{12}$ does support the conclusion that Moby is a mammal, and also the conclusion that mammals are air-breathers. But we can't put these two ideas together in $\Gamma_{12}$ to conclude that Moby is an air-breather. In $\Gamma_{12}$, the argument showing that mammals are air-breathers depends on their being land-dwellers. Therefore, we shouldn't be able to apply this general conclusion about mammals to Moby, since the general conclusion holds of mammals only in virtue of their being land-dwellers, and we know of Moby in particular that he is not a land-dweller. It is different in $\Gamma_{13}$. Here, the fact that mammals are air-breathers no longer depends solely on the fact that they are land-dwellers. According to $\Gamma_{13}$, mammals would be air-breathers even if they weren't land-dwellers. Therefore, the fact that Moby in particular isn't a land-dweller shouldn't interfere in $\Gamma_{13}$ with the general argument that, since he is a mammal, he is an air-breather.

\subsection{Intersections of credulous extensions}

We mentioned in Section 3 that the credulous (or belief-hungry) approach tends to associate with nets containing compound conflicting paths a number of different consistent extensions, or fixed points. It is tempting, therefore, to suppose that the set of paths permitted by a given net under the present skeptical analysis might simply be the intersection of the various extensions associated with that net according to the credulous analysis provided by [22]. However, nets like $\Gamma_{14}$ (Fig. 14)-which have the topology of nested Nixon Diamonds-show that this is not so. In this case, we have $\Gamma_{14} p a \rightarrow p+q$. The potentially conflicting path $a \rightarrow s \rightarrow t \rightarrow q$ poses no problem; this path is not permitted, since its initial segment $a \rightarrow s \rightarrow t$ is itself neutralized by the path

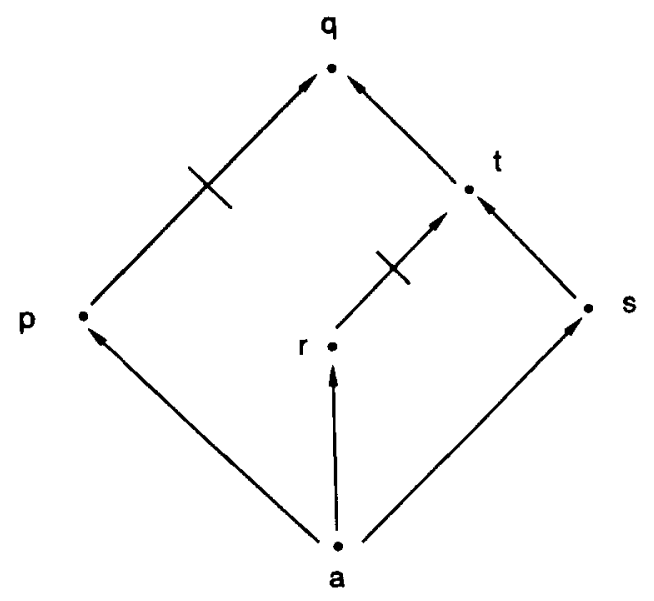

Fig. 14. $\Gamma_{14}$. 
$a \rightarrow r \nrightarrow t$. But the path $a \rightarrow p \rightarrow q$ isn't contained in all the credulous extensions associated with this net; some contain instead the path $a \rightarrow s \rightarrow t \rightarrow q$.

\subsection{Decoupling}

In inheritance reasoners that construct inference paths through backward chaining, such as that of Touretzky [22], conclusions about items in a network depend on conclusions about their immediate superiors. According to Touretzky's theory, for example, a path of the form $x \rightarrow u \rightarrow \sigma \rightarrow y$ will belong to an extension only if the path $u \rightarrow \sigma \rightarrow y$ also belongs to that extension; a path of the form $x \rightarrow u \rightarrow \sigma \nrightarrow y$ will belong to an extension only if $u \rightarrow \sigma \rightarrow y$ does.

As the net $\Gamma_{15}$ (Fig. 15) shows, our analysis allows items in a network to be decoupled from their immediate superiors, in the sense that it allows particular items to possess properties possessed by none of their immediate superiors. Here, we have $\Gamma_{15} p a \rightarrow p \rightarrow q \rightarrow s$. The potentially conflicting path $a \rightarrow p \rightarrow r \nrightarrow s$ poses no problem since its compound initial segment $a \rightarrow p \rightarrow r$ conflicts with the direct link $a \rightarrow r$. On the other hand, though $\Gamma_{15}$ permits $a \rightarrow p \rightarrow q \rightarrow s$, and so supports the statement $a \rightarrow s$, the net does not permit the path $p \rightarrow q \rightarrow s$, and indeed does not support the statement $p \rightarrow s$.

This kind of decoupling can seem a bit anomalous if one's ideas about inheritance reasoning are conditioned by the top-down or "property flow" approach, according to which individuals are supposed to inherit their properties strictly in virtue of belonging to certain classes of things--their ancestors in the network-which possess those properties. The problem is that, while $\Gamma_{15}$ supports the statement that the individual $a$ is an $s$, it is unclear how $a$ could have inherited this property. After all, the only immediate ancestor of $a$ in the network is the node $p$. According to the top-down approach, then, $a$ must have inherited all the positive properties it does inherit simply in virtue of being a $p$; if it possess any particular property, such as being an $s$, this could only be due to the fact that $p$ 's possess that property. But as we have seen, $\Gamma_{15}$ doesn't support the statement that $p$ 's are $s$ 's.

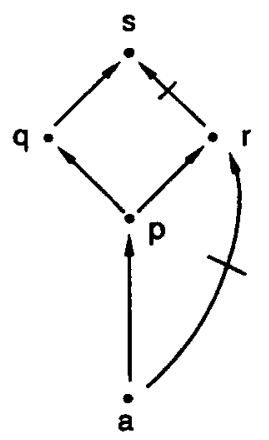

Fig. 15. $\Gamma_{15}$. 
Against the background of the bottom-up or "argument construction" view of inheritance reasoning, however, the situation presented by this example is perfectly coherent. Since $\Gamma_{15}$ contains the materials for constructing unpreempted, compound arguments enabling both the conclusion that $p$ 's are $s$ 's and the conclusion that $p$ 's are not $s$ 's, our broadly skeptical point of view forces us to withhold judgment, endorsing neither of these conclusions. The individual $a$, though, is a particular $p$ for which the general kind of argument enabling the conclusion that $p$ 's are not $s$ 's is blocked: that argument depends on the information that $p$ 's are $r$ 's, but $\Gamma_{15}$ tells us explicitly that $a$ is not an $r$. Since the general argument that $p$ 's are not $s$ 's is explicitly blocked for this particular indiviudal, then, it cannot conflict in the case of $a$ with the argument that $p$ 's are $s$ 's; so we conclude that $a$ is an $s$.

\section{A Hybrid Inference Algorithm}

Inheritance networks are attractive as formalisms for knowledge representation because they allow information to be organized in such a way that certain important kinds of inference can be carried out through efficient graphsearching techniques. In particular, ever since their inception in the work of Quillian [16], network representations have been associated with parallel inference schemes. This tendency culminated in the NETL system of Fahlman [5], which combined a nonmonotonic network representation languge with a massively parallel reasoning architecture, known as a Parallel Marker Propagation Machine (PMPM). Unfortunately, the NETL representation language was never provided with a clear semantics of its own, independent of the associated inference algorithms; and it turned out, because of their treatment of exceptions, that these algorithms often led to anomalous results [7]. Once a satisfactory semantic account was developed for inheritance networks with exceptions-initially, with the work of Etherington [4] and Touretzky [22]--it soon became clear that the kinds of inferences appropriate to these networks could not be carried out through purely parallel reasoning.

In this section, after reviewing Fahlman's PMPM architecture, we present a hybrid (parallel-serial) inference algorithm that reasons in accord with the definition of inheritance presented here. The algorithm is designed to exploit the parallelism of the PMPM to the greatest extent possible, resorting to serial reasoning only when necessary.

\subsection{Parallel marker propagation machines}

A PMPM is an automaton composed of active elements that play the roles of nodes and links in a graph. Each element has a small number of internal states (marker bits, which can be on or off, representing the presence or absence of markers), and a limited ability to communicate information to the elements to which it is connected. The nodes and links in a PMPM are responsive to 
various marker propagation commands, each of which directs the assignment of markers to particular nodes, often by "propagating" them from one node to another through the intervening links. PMPMs are SIMD (Single Instruction stream, Multiple Data stream) machines: marker propagation commands are broadcast globally to all elements and executed in parallel by the elements to which they apply. Parallel marker propagation algorithms can be described as sequences of marker propagation commands; the result of executing such an algorithm in a particular net is a coloring - a static assignment of marker bits to nodes-that is used to convey some information about the net.

The notation used here for specifying marker propagation commands is a slight extension of the one defined in Touretzky [22]. Commands may be either conditional or unconditional. Unconditional commands are executed by all elements regardless of their current state. The unconditional command clear $\left[\mathrm{M}_{1}\right]$, for instance, causes all elements to clear marker bit $\mathrm{M}_{1}$. Conditional commands are more common. The command,

$$
\text { link-type[" } \left.\rightarrow \text { "], on-tail[ } M_{1}\right], \text { off-head }\left[M_{1}\right] \Rightarrow \operatorname{set-head}\left[M_{1}\right],
$$

would be executed by any element meeting the conditions on the left-hand side of the arrow: if the element represents a link of type " $\rightarrow$ ", the node at its tail bears the marker $M_{1}$, and the node at its head does not bear marker $M_{1}$, then the link will perform the action specified on the right-hand side of the arrow, marking the node at its head with $\mathrm{M}_{1}$.

Looping is accomplished with a simple loop body endloop construct, which repeats the commands in the body of the loop until no conditional command appearing in the body has its left-hand side satisfied. The following loop, for example, propagates the marker $M_{1}$ up " $\rightarrow$ " links, thereby computing the transitive closure of the " $\rightarrow$ " relation. The loop terminates when all eligible nodes have been marked with $M_{1}$. (The off-head[ $\left.M_{1}\right]$ condition assures that nodes already marked with $M_{1}$ are not eligible to be marked on subsequent iterations.)

$$
\begin{aligned}
& \text { loop } \\
& \left.\quad \text { link-type [" } \rightarrow \text { "'], on-tail[ }\left[M_{1}\right] \text {, off-head }\left[M_{1}\right] \Rightarrow \text { set-head[ } M_{1}\right] \\
& \text { endloop }
\end{aligned}
$$

It is also possible to address particular nodes by name using conditional commands. The node $x$ would be selected by placing the restriction name $[x]$ on the left-hand side of the conditional arrow; only the element representing that node would then respond. This technique is used to select and mark an initial node at the beginning of certain marker propagation procedures. For example, the procedure below would compute the transitive closure of the " $\rightarrow$ " relation starting at a given node $x$, marking the nodes in the resulting set with $M_{1}$. 


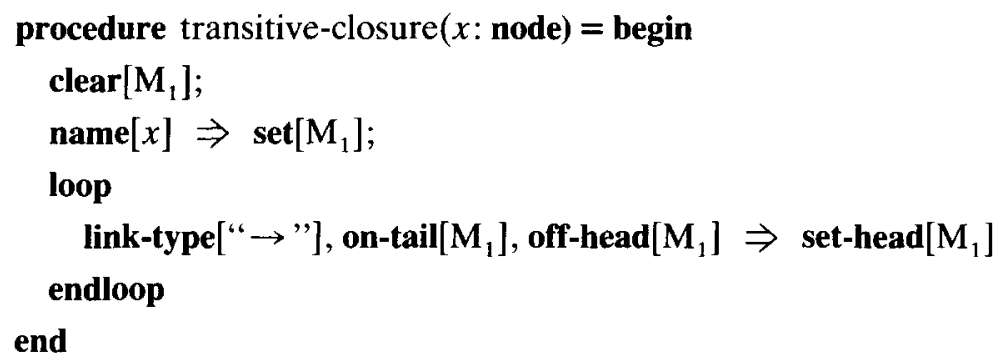

The set of conditions on the left-hand side of the conditional arrow are always treated conjunctively. Many conditions accept multiple arguments, which also are treated conjunctively; for example, on-tail $\left[M_{1}, M_{2}\right]$ is satisfied by a link element only if it satisfies both on-tail $\left[\mathrm{M}_{1}\right]$ and on-tail $\left[\mathrm{M}_{2}\right]$. It is possible, however, to specify disjunctions of markers on the left-hand side, by using a different set of multi-argument commands: the link-type condition and all conditions beginning with "any-" are disjunctive. For example, the command any-on-tail $\left[M_{1}, M_{2}\right]$ calls those link elements that satisfy either on$\operatorname{tail}\left[\mathrm{M}_{1}\right]$ or on-tail[ $\left.\mathrm{M}_{2}\right]$.

A parallel marker propagation machine is controlled by a host computer that broadcasts commands to all the individual elements, where they are executed in synchrony. The extension we have made here to the PMPM algorithm notation-a loop of the form for var in 〈parallel-condition〉 do body-doesn't really affect the parallel capabilities of the machine at all, but involves only statements to be executed by the host. The point of the extension is to allow elements to be processed serially when necessary. The parallel-condition specifies a conditional test, equivalent to the left-hand side of a rule, that is broadcast to all elements. Elements satisfying the condition are then identified by the host computer, using some addressing mechanism which we won't go into here (see Fahlman [5] for details), and processed serially.

\subsection{Skeptical inheritance on a PMPM}

Because each of the computing elements of a PMPM can represent only a small, fixed number of marker bits, it isn't possible to use a PMPM algorithm to compute, all at once, the entire theory of a net: for a net with $N$ nodes, that would require each node to carry at least $2 N$ marker bits. Moreover, since it is only under rare circumstances that a user would actually be interested in knowing the entire theory of a network, it would be unwise in any case to invest the computational resources necessary for computing the full theory. In the general case, the user comes to a database with a particular query in mind: he wants to know whether the database supports a particular statement, or its negation. Therefore, what we define here is a query procedure, query $(x, y)$, which is able to determine, for any net $\Gamma$ and nodes $x$ and $y$, whether $\Gamma$ 
supports the statement $x \rightarrow y$, the statement $x \rightarrow y$, neither statement, or both.

The procedure we define is economical. It scans only that portion of the network directly relevant to a particular query. It uses only fourteen markers all told, and only the two markers $M_{T}$ and $M_{F}$ to represent the result of the query. As a result of performing the query $(x, y)$ in the net $\Gamma$, the marker $\mathrm{M}_{\mathrm{T}}$ will be present on the node $y$ iff $\Gamma$ supports $x \rightarrow y$, and marker $\mathrm{M}_{\mathrm{F}}$ will be present on the node $y$ iff $\Gamma$ supports $x \rightarrow y$.

\subsubsection{Degree ${ }^{x, y}$}

In Section 4, we ordered the paths in a given net by degree, and then proceeded to define the permission relation through an induction on the degree of a path in a net. In that context-where definition, not implementation, was the issue - the ordering by degree was appropriate, since it is a particularly simple ordering, and it is adequate, in the sense that all the paths that could possibly be relevant to a given path are assigned a lesser degree.

In the present context, however, since implementation is itself the issue, the ordering by degree is no longer quite so appropriate. For reasons of efficiency, we would like our query procedure to examine the minimum of paths necessary to decide whether a particular statement is supported by a net; but if it were to sort through the paths in the net by degree, in addition to all the paths relevant to the query, the query procedure would wind up considering a number of irrelevant paths as well. For example, in the net $\Gamma_{16}$ (Fig. 16), both the paths $x \rightarrow t \rightarrow u$ and the path $x \rightarrow p \rightarrow q \rightarrow r$ are assigned a lower degree than the path $x \rightarrow z \rightarrow y$. Since it is obvious from the structure of the network, however,

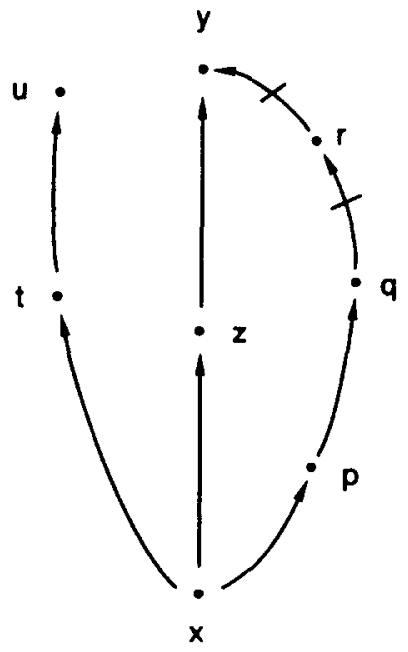

Fig. 16. $\Gamma_{16}$.

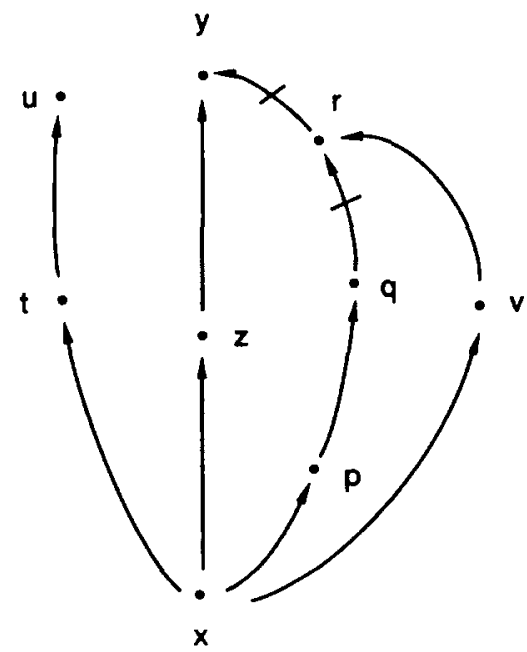

Fig. 17. $\Gamma_{17}$. 
that neither of these paths could possibly have an effect on the path $x \rightarrow z \rightarrow y$, it would be wasteful for the procedure query $(x, y)$ even to consider them. In order to implement our query procedure efficiently, we need to define another ordering of the paths in a net, which differs from the standard by degree in considering only the paths relevant to a particular query.

As a first step, we specify the restriction of a net $\Gamma$ with respect to the query $(x, y)$. Intuitively, this query-restricted network, written $\Gamma^{x, y}$, is supposed to represent the subgraph of $\Gamma$ which it is necessary to examine in order to determine whether $\Gamma$ supports either of the statements $x \rightarrow y$ or $x \rightarrow y$. We capture the notion formally by specifying that $\Gamma^{x, y}$ is the minimal set containing (i) every link on every path in $\Gamma$ from $x$ to $y$, as well as (ii) every link on every path in $\Gamma$ from $x$ to $w$, for all nodes $w$ occurring in $\Gamma^{x, y}$. For example, we have $\Gamma_{16}^{x, y}=\{x \rightarrow z, z \rightarrow y\}$, since $x \rightarrow z \rightarrow y$ is the only path in $\Gamma_{16}$ from $x$ to $y$, and there are no other paths in $\Gamma_{16}$ from $x$ to any of the nodes on this path. However, $\Gamma_{17}^{x, y}$ includes, as it should, every link in $\Gamma_{17}$ except for the two links $x \rightarrow t$ and $t \rightarrow u$ (see Fig. 17). Since $\Gamma_{17}$ contains the additional path $x \rightarrow v \rightarrow r \rightarrow y$, the path $x \rightarrow p \rightarrow q \nrightarrow r$, which was contained in $\Gamma_{16}$ but irrelevant there to query $(x, y)$, is now relevant to the query; so the links in this path must be contained in $\Gamma_{17}^{x, y}$.

Once we have defined the query-restricted net $\Gamma^{x, y}$ in this way, it is easy to show that, for any path $\sigma$ in $\Gamma^{x, y}, \Gamma p \sigma$ iff $\Gamma^{x, y} p \sigma$. It is then natural to define the degree of a path $\sigma$ in a net $\Gamma$ with respect to the query $(x, y)$-written $\operatorname{deg}_{\Gamma}^{x, y}(\sigma)$-as the degree of the path $\sigma$ in the suitably restricted net $\Gamma^{x, y}$. Formally, we take

$$
\operatorname{deg}_{\Gamma}^{x, y}(\sigma)= \begin{cases}\operatorname{deg}_{\Gamma^{x, y}}(\sigma), & \text { if } \sigma \text { is a path in } \Gamma^{x, y}, \\ \text { undefined, } & \text { otherwise. }\end{cases}
$$

In order to distinguish it from the familiar (Section 4.1) notion of degree, we refer to this new, query-restricted notion as degree ${ }^{x, y}$. Evidently, the procedure query $(x, y)$ can accurately determine whether a net $\Gamma$ supports $x \rightarrow y$ or $x \rightarrow y$ while limiting its attention to $\Gamma^{x, y}$ : it need only consider those paths in a net

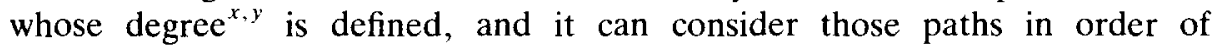
increasing degree ${ }^{x, y}$.

For convenience, we will also refer in what follows to the degree ${ }^{x, y}$ of a node; where $w$ is a node, the degree ${ }^{x, y}$ in $\Gamma$ of $w$ is defined as the degree ${ }^{x, y}$ of any path in $\Gamma$ from $x$ to $w$. (Of course, all such paths have the same degree $^{x, y}$.)

\subsubsection{The algorithm}

We can now describe query $(x, y)$, our PMPM algorithm for responding to queries in accord with the definition of skeptical inheritance presented here. We begin with the two subprocedures trim-for-query $(x, y)$ and select-nextdegree (); after that, we present the bulk of query $(x, y)$ itself, with the exception of the subprocedure check-preemption $(c)$, which is described last of all. 
The procedure trim-for-query $(x, y)$ trims the network $\Gamma$ so that only the query-restricted $\Gamma^{x, y}$ will be considered in future processing. It begins in lines 3 and 4 by marking $x$ with $M_{x}$ and $y$ with $M_{y}$. In the loop at lines 5-8, it propagates the marker $\mathbf{M}_{1}$ up positive paths in $\Gamma$ from $x$. If there is a path in $\Gamma$, positive or negative, from $x$ to $y$, it marks $y$ with $M_{2}$ in lines 9-10. Finally, in the loop at lines 11-14, the procedure propagates $M_{2}$ down both " $\rightarrow$ " links and " $\rightarrow$ " links in $\Gamma$ into those nodes already marked with $\mathbf{M}_{1}$.

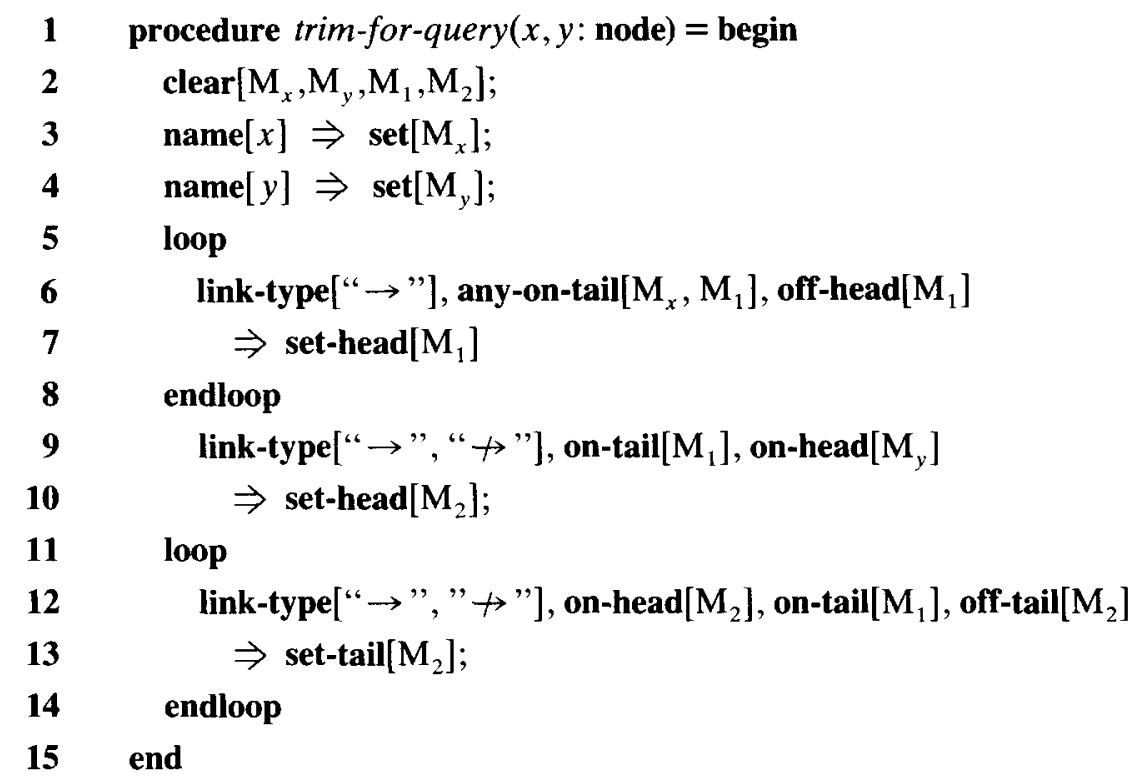

The overall effect of carrying out this procedure in a net $\Gamma$ is to mark each node in $\Gamma^{x, y}$ (with the exception of $x$ itself) with the marker $\mathbf{M}_{2}$, a fact recorded in the following lemma. In future processing, we then manage to ignore irrelevant paths by restricting certain commands so that they apply only to nodes marked with $\mathrm{M}_{2}$.

Lemma 6.1. As a result of running the procedure trim-for-query $(x, y)$ in $\Gamma$, each node $w$ occurring in $\Gamma$ will be marked with $\mathrm{M}_{2}$ iff $w \neq x$ and $w$ occurs in $\Gamma^{x, y}$.

Proof. Trim-for-query $(x, y)$ begins in lines $5-8$ by marking the nodes on positive paths in $\Gamma$ from $x$, except for $x$ itself, with $\mathrm{M}_{1}$. It is clear that $y$ itself will be marked with $\mathrm{M}_{2}$ in lines 9-10 iff there is a path from $x$ to $y$ in $\Gamma$. Now suppose that not all nodes occurring in $\Gamma^{x, y}$ are marked with $\mathrm{M}_{2}$; in particular, let $w$ be a node of maximal degree ${ }^{x, y}$ in $\Gamma^{x, y}$ that is not marked with $\mathbf{M}_{2}$. Since $w$ occurs in $\Gamma^{x, y}, \Gamma$ must contain a path either of the form $x \rightarrow \tau \rightarrow w \rightarrow z$ or of 
the form $x \rightarrow \tau \rightarrow w \nrightarrow z$, such that $z$ occurs in $\Gamma^{x, y}$; since $w$ is maximal, $z$ will be marked with $\mathbf{M}_{2}$. Since $w$ is on a positive path from $x, w$ will have to be marked with $M_{1}$; so $w$ will be marked with $M_{2}$ in the loop at lines 11-14, which contradicts our assumption. Thus, all nodes occurring in $\Gamma^{x, y}$ will be marked with $\mathrm{M}_{2}$. Further, since only nodes that are on paths from $x$ to a node with $\mathrm{M}_{2}$ will be marked with $\mathrm{M}_{2}$, all nodes marked with $\mathrm{M}_{2}$ will occur in $\Gamma^{x, y}$.

The procedure select-next-degree() will be called each time through the main loop of the procedure query $(x, y)$ to mark the nodes of degree $_{x, y} n$.

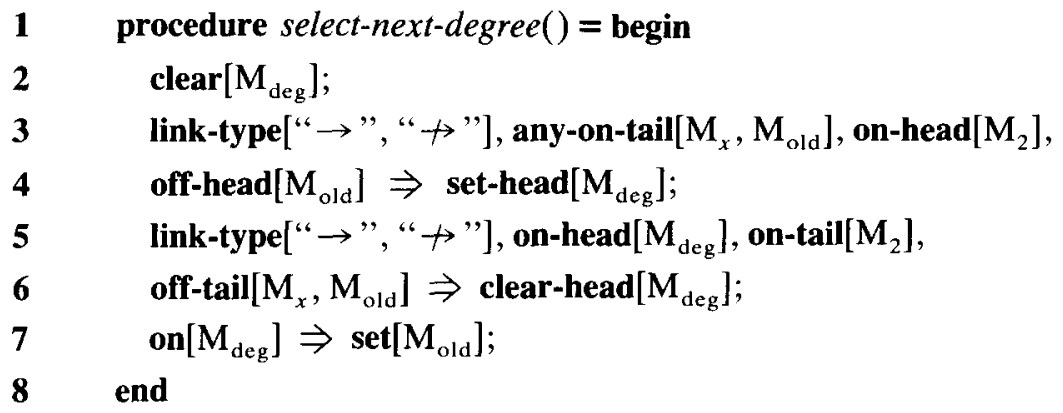

The effect of this procedure, in the context in which it occurs in query $(x, y)$, is recorded in the following lemma.

Lemma 6.2. After executing trim-for-query $(x, y)$ and clearing $\mathrm{M}_{\text {old }}$ in a network $\Gamma$, the nth call to select-next-degree() leaves exactly the nodes of degree ${ }^{x, y} n$ in $\Gamma$ marked with $\mathrm{M}_{\mathrm{deg}}$, and exactly the nodes of degree ${ }^{x, y}$ less than or equal to $n$ in $\Gamma$ marked with $\mathbf{M}_{\text {old }}$.

Proof. Nodes of degree ${ }^{x, y} 1$ will be connected to $x$ in $\Gamma^{x, y}$ by direct links, and only direct links. It is obvious that the first time select-next-degree() is called, it will mark all such nodes with $\mathbf{M}_{\text {deg }}$ in lines 3-4; the command on lines 5-6 can have no effect, since it applies only to links not emanating from $x$; and then at line 7 , the nodes marked with $\mathbf{M}_{\text {deg }}$ will be marked also with $\mathbf{M}_{\text {old }}$. Thus the theorem is true for $n=1$.

For induction, suppose the theorem holds for all calls prior to the $n$ th. The nodes of degree ${ }^{x, y} n$ are just those nodes $w$ for which (i) $\Gamma^{x, y}$ contains either of the links $u \rightarrow w$ or $u \nrightarrow w$ with $u$ a node of degree ${ }^{x, y} n-1$; and (ii) $\Gamma^{x, y}$ contains no links $v \rightarrow y$ or $v \nrightarrow y$ with $v$ a node of degree ${ }^{x, y}$ greater than or equal to $n$. Given the inductive hypothesis, then, the procedure select-nextdegree() behaves as follows on its $n$th call. First, in line 2 , it clears the marker $\mathbf{M}_{\mathrm{deg}}$ from nodes of degree ${ }^{x, y} n$. Next, in lines $3-4$, it places $\mathbf{M}_{\mathrm{deg}}$ on exactly 
those nodes $w$ satisfying (i) - since, by hypothesis, any such node $u$ will already be marked with $\mathrm{M}_{\text {old }}, w$ will of course be marked with $\mathrm{M}_{2}$, but $w$ will not yet be marked with $\mathbf{M}_{\text {old }}$. Then, in lines 5-6, it clears $\mathbf{M}_{\text {deg }}$ from all those nodes $w$ satisfying (i) except those also satisfying (ii)-since, if $w$ fails to satisfy (ii), any such node $v$ will of course be marked with $\mathbf{M}_{2}$ and will by hypothesis be marked neither with $M_{x}$ nor $M_{\text {old }}$. Finally, in line 7, the procedure marks the nodes displaying $M_{\text {deg }}$ also with $M_{\text {old }}$. Thus, if the theorem is true for the all calls prior to the $n$ th, it is true also for the $n$th call.

At this point, we can describe query $(x, y)$ itself, our main inference algorithm.

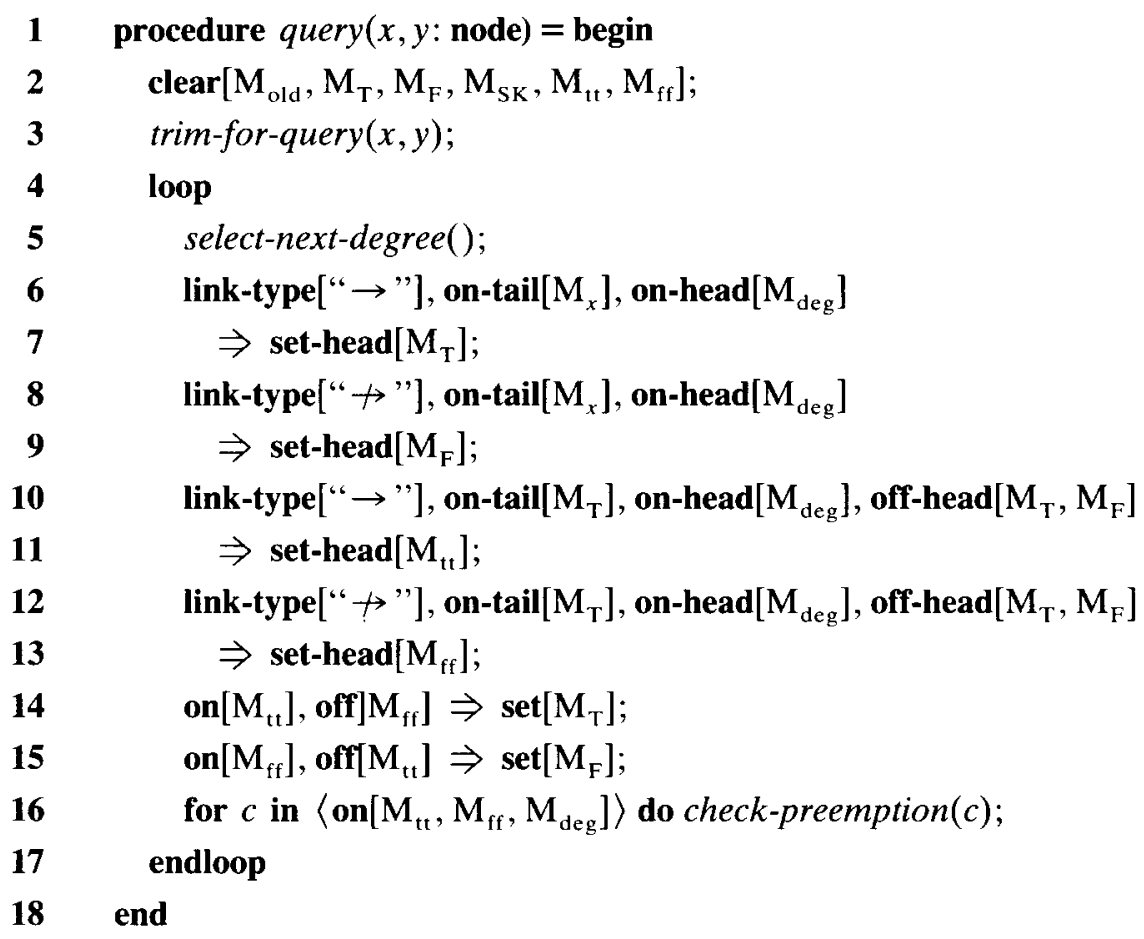

The procedure begins on line 2 by clearing the result marks $M_{T}, M_{F}$, and $M_{S K}$, as well as the auxiliary marks $\mathrm{M}_{\mathrm{tt}}$, and $\mathrm{M}_{\mathrm{ff}}$, and then calling trim-forquery $(x, y)$ on line 3 to mark all the relevant nodes with $\mathrm{M}_{2}$. Then the main loop (lines 4-17) is entered. Through the calls to select-next-degree() on line 5, nodes are processed in order of increasing degree: during each iteration $n$ of the loop, the nodes of exactly degree ${ }^{x, y} n$ are selected and marked for processing with $\mathbf{M}_{\text {deg }}$. If there are direct links from $x$ to $w$, where $x$ is a node of degree $^{x, y} n$, lines 6-9 mark $w$ with $\mathrm{M}_{\mathrm{T}}$ or $\mathrm{M}_{\mathrm{F}}$, as appropriate. The real work begins on lines $10-11$. Here, each node $w$ of appropriate degree $^{x, y}$ is given the 
auxiliary marker $\mathrm{M}_{\mathrm{t}}$ if it is connected by a " $\rightarrow$ " link to some node $u$ already marked with $\mathrm{M}_{\mathrm{T}}$. Likewise, in lines $10-11, w$ is marked with $\mathrm{M}_{\mathrm{ff}}$ if it is connected by a " $\nrightarrow$ " link to a node $v$ already marked with $\mathrm{M}_{\mathrm{T}}$. These auxiliary markers indicate tentative conclusions. If $w$ is marked with $M_{t t}$ in lines 8-9, but not with $\mathrm{M}_{\mathrm{ff}}$ in lines 12-13, there is tentative evidence for thinking that the statement $x \rightarrow w$ should be supported, and no evidence to the contrary; therefore, the node will be marked with $\mathrm{M}_{\mathrm{T}}$ in line 14. Likewise, if $w$ is marked with $\mathrm{M}_{\mathrm{ff}}$ but not $\mathrm{M}_{\mathrm{tt}}$, it will be marked in line 15 with $\mathrm{M}_{\mathrm{F}}$. However, some nodes may be marked with both $\mathrm{M}_{\mathrm{tt}}$ and $\mathrm{M}_{\mathrm{ff}}$ : if $w$ is such a node there is tentative reason to think $x \rightarrow w$ is supported, as well as a tentative reason for thinking that $x+w$ is supported. For each of these "conflicted" nodes, we must then call the following check-preemption procedure individually, in line 16 , to determine whether either of the conflicting tentative arguments are preempted.

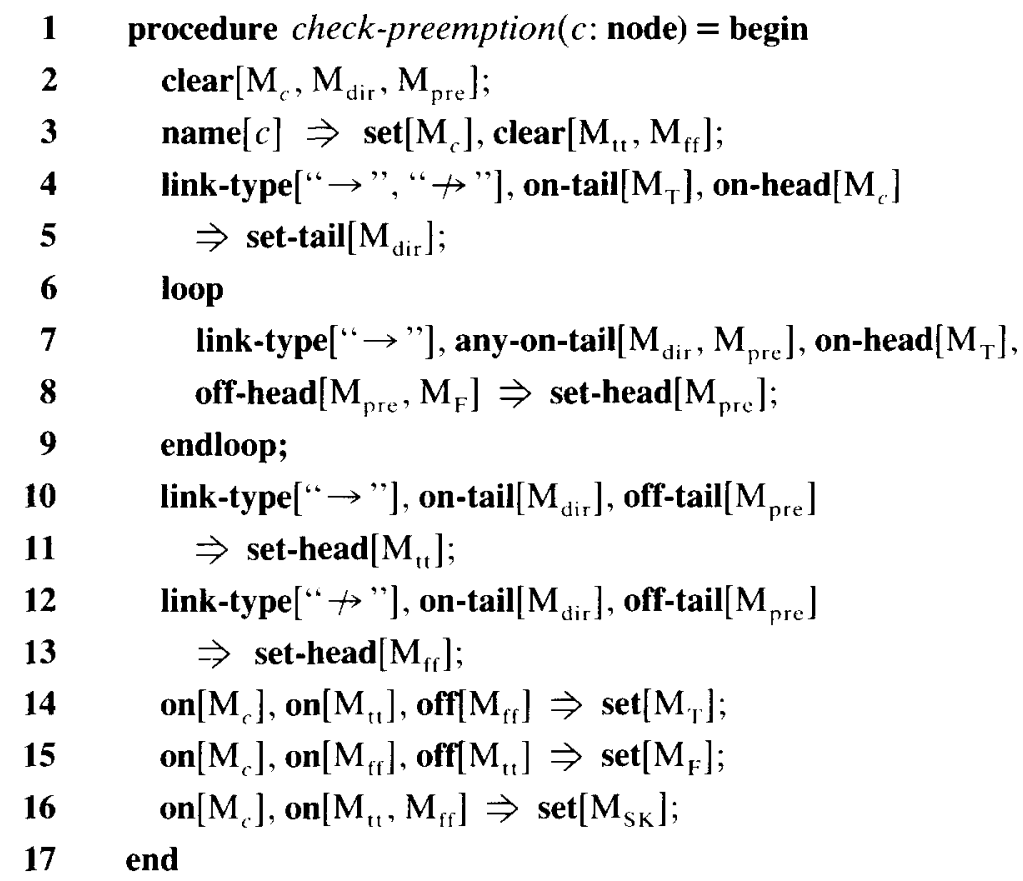

Where $c$ is such a conflicted node, the procedure check-preemption $(c)$ marks nodes with direct links to $c$ with $\mathrm{M}_{\mathrm{dir}}$ on lines 4-5. It then marks preempted nodes, in the loop at lines 6-9, by propagating $M_{\text {pre }}$ upwards from nodes with $M_{\mathrm{dir}}$. In lines 10-13, the tentative markers $\mathrm{M}_{\mathrm{tt}}$ and $\mathrm{M}_{\mathrm{ft}}$, which were deleted from $c$ in line 3, are reset only if they were propagated from nodes which are not marked as preempted. If only one of these two tentative markers is reset, it must be that the tentative conflicting evidence was preempted; so $c$ is marked 
in lines 14-15 either with $\mathrm{M}_{\mathrm{T}}$ or with $\mathrm{M}_{\mathrm{F}}$, as appropriate. If both of the tentative markers are reset, then neither of the conflicting tentative arguments is preempted. In accord with our general skeptical viewpoint, therefore, these paths neutralize each other; so the node $c$ is marked in line 16 with $\mathrm{M}_{\mathrm{SK}}$.

\subsection{Correctness of the algorithm}

The PMPM algorithm we have described in this section can be proved to be correct-that is, both sound and complete-with respect to the inheritance definition from Section 4.

Theorem 6.3. As a result of executing the procedure query $(x, y)$ in a net $\Gamma$, the node $y$ will be marked with $\mathrm{M}_{\mathrm{T}}$ iff $\Gamma$ supports $x \rightarrow y$, and with $\mathrm{M}_{\mathrm{F}}$ iff $\Gamma$ supports $x \rightarrow y$.

This theorem follows at once from the following lemma, along with the observation that no command in query $(x, y)$ ever deletes either of the markers $\mathrm{M}_{\mathrm{T}}$ or $\mathrm{M}_{\mathrm{F}}$.

Lemma 6.4. Suppose procedure query $(x, y)$ is executed in $\Gamma$; let $w$ be a node of degree ${ }^{x, y} n$ in $\Gamma$. Then $w$ will be marked with $\mathrm{M}_{\mathrm{T}}$ during the nth iteration of the main loop in query $(x, y)$ iff $\Gamma$ supports $x \rightarrow w$, and $w$ will be marked with $\mathbf{M}_{\mathrm{F}}$ during the nth iteration of the main loop in query $(x, y)$ iff $\Gamma$ supports $x \rightarrow w$.

Proof. First, let the degree ${ }^{x, y}$ of $w$ be 1 , so that $\Gamma$ contains direct links, and only direct links, from $x$ to $w$. Suppose $x \rightarrow w \in \Gamma$. Then of course $\Gamma$ supports $x \rightarrow w$. Lemma 6.2 tells us that, on the first time through its main loop, query $(x, y)$ marks $w$ with $\mathrm{M}_{\text {deg }}$ in line 5; then $w$ is marked in lines 6-7 with $\mathrm{M}_{\mathrm{T}}$. Similar reasoning shows us that the theorem holds also when $x \rightarrow w \in \Gamma$. Therefore, the theorem is true when $w$ is a node of degree ${ }^{x, y} 1$. Assuming the theorem is true for nodes of degree ${ }^{x, y}$ less than $n$, we show that it's true also for the node $w$, where $w$ is of degree ${ }^{x, y} n$. The induction proceeds in four parts.

Part 1: Completeness for positive paths. Suppose $\Gamma$ supports $x \rightarrow w$. If $x \rightarrow w \in \Gamma$, Lemma 6.2 tells us that $w$ will be marked with $\mathrm{M}_{\text {deg }}$ by the time the $n$th pass through the main loop of query $(x, y)$ reaches line 6 ; it will therefore be marked with $\mathrm{M}_{\mathrm{T}}$ in lines 6-7. If $x \rightarrow w \notin \Gamma$, then $\Gamma$ must permit some path of the form $x \rightarrow \sigma_{1} \rightarrow u \rightarrow w$. By II.1(a) of the inheritance definition, we know $\Gamma \triangleright x \rightarrow \sigma_{1} \rightarrow u$; so by inductive hypothesis, query $(x, y)$ marks $u$ with $\mathrm{M}_{\mathrm{T}}$ prior to the $n$th iteration of the main loop. By II.1(b), we know $u \rightarrow w \in \Gamma$. Therefore, on the $n$th iteration of the main loop, when Lemma 6.2 tells us that the node $w$ is marked with $\mathrm{M}_{\mathrm{deg}}, w$ will be marked in lines $10-11$ with $\mathrm{M}_{\mathrm{tt}}$. If there is no node $v$ such that $v \rightarrow w \in \Gamma$ and $\Gamma \ngtr x \rightarrow \tau \rightarrow v$, we can see using the inductive hypotheses that the node $w$ cannot be marked with $\mathrm{M}_{\mathrm{ff}}$ in lines 
12-13. It will therefore be marked with $\mathrm{M}_{\mathrm{T}}$ at line 14 . On the other hand, if there is a node $v$ such that $v \nrightarrow w \in \Gamma$ and $\Gamma \triangleright x \rightarrow \tau \rightarrow v$, we can see using the inductive hypothesis that the node $w$ will be marked with $\mathrm{M}_{\mathrm{ff}}$ in lines 12-13; it will therefore satisfy the condition in the for loop at line 16 , and so checkpreemption $(c)$ will have to be called, with $c=w$.

For any such node $v$, clause II.1(d) tells us that there must be a $z$ such that $z \rightarrow w \in \Gamma$ and either $z=x$ or $\Gamma \triangleright x \rightarrow \tau_{1} \rightarrow z \rightarrow \tau_{2} \rightarrow v$. There may, of course, be many nodes $z$ satisfying this condition: let $z^{\prime}$ be one of the minimal degree $^{x, y}$. In line 3 of check-preemption $(c)$, the markers $\mathrm{M}_{\mathrm{tt}}$ and $\mathrm{M}_{\mathrm{ff}}$ are cleared from $w$, and $\mathrm{M}_{c}$ is set. In lines $4-5$, the marker $\mathrm{M}_{\mathrm{dir}}$-indicating a direct link to $w$-is set on both the nodes $v$ and $z^{\prime}$. Since $\Gamma \triangleright x \rightarrow \tau_{1} \rightarrow z^{\prime} \rightarrow \tau_{2} \rightarrow v$, the inductive hypothesis tells us that every nodes on this path must display the marker $\mathrm{M}_{\mathrm{T}}$ by the time we enter the $n$th iteration of the main query $(x, y)$ loop. Therefore, the loop in lines 6-9 of check-preemption $(c)$ will propagate $\mathrm{M}_{\text {pre }}$ up this path, beginning with the node immediately after $z^{\prime}$ and continuing all the way to $v$ itself. However, since $z^{\prime}$ is minimal, there are no nodes on permitted paths from $x$ to $z^{\prime}$ with direct links to $w$; so we can see using the inductive hypothesis that $z^{\prime}$ will not be marked with $\mathrm{M}_{\mathrm{pre}}$. Therefore, $w$ will be marked with $\mathbf{M}_{\mathrm{tt}}$ in lines 10-11 of check-preemption $(c)$, but not with $M_{\mathrm{ff}}$ in lines 12-13; and so on line 14, $w$ will be marked with $\mathrm{M}_{\mathrm{T}}$.

Part 2: Soundness for positive paths. Suppose that query $(x, y)$ marks $w$ with $\mathrm{M}_{\mathrm{T}}$ on the $n$th iteration of the main loop. There are only three commands in the loop that could result in w's being marked with $\mathrm{M}_{\mathrm{T}}$ : (i) the command in lines 6-7 of query $(x, y)$, (ii) the command in line 14 of query $(x, y)$, and (iii) the command in line 14 of check-preemption $(c)$. We examine them in turn, showing that no matter how $\mathrm{M}_{\mathrm{T}}$ is assigned to $w$, it must turn out that $\Gamma$ supports $x \rightarrow w$.

(i) If $w$ is marked with $\mathrm{M}_{\mathrm{T}}$ in lines 6-7 of query $(x, y)$, it must be that $x \rightarrow w \in \Gamma$. Hence, $\Gamma$ supports $x \rightarrow w$.

(ii) Suppose $w$ is marked with $\mathrm{M}_{\mathrm{T}}$ in line 14 of query $(x, y)$. Then $w$ must have been marked with $M_{t t}$ in lines 10-11. Looking at lines 10-11, we can see therefore, that there must be some node $u$ with $u \rightarrow w \in \Gamma$ such that query $(x, y)$ marks $u$ with $\mathrm{M}_{\mathrm{T}}$. By inductive hypothesis, then, $\Gamma \ngtr x \rightarrow \sigma_{1} \rightarrow u$, for some path $\sigma_{1}$. This tells us that clauses II.1(a) and II.1(b) of the inheritance definition are satisfied. Moreover, clause II.1(c) is satisfied as well: we know that $x \nrightarrow w \notin \Gamma$, since if $x \rightarrow w \in \Gamma$, then $w$ would have been marked with $\mathrm{M}_{\mathrm{F}}$ at line 8 , and so $w$ could not have been marked with $\mathrm{M}_{\mathrm{tt}}$ in lines 10-11. Finally, suppose there were some node $v$ such that $\Gamma \ngtr x \rightarrow \tau \rightarrow v$ and $v \rightarrow y \in \Gamma$. By inductive hypothesis, query $(x, y)$ would already have marked $v$ with $\mathrm{M}_{\mathrm{T}}$ on a previous iteration of the loop. The node $w$ would then be marked with $\mathbf{M}_{\mathrm{ff}}$ in lines 12-13 of the current iteration, and so $w$ could not be marked with $\mathrm{M}_{\mathrm{T}}$ at line 14, contrary to our supposition. Hence, there can be no such node $v$, and clause II.1(d) is vacuously true. Therefore, since the clauses II.1(a) through II.1(d) are satisfied, $\Gamma \triangleright x \rightarrow \sigma_{1} \rightarrow u \rightarrow w$, and so $\Gamma$ supports $x \rightarrow w$. 
(iii) Suppose $w$ is marked with $\mathrm{M}_{\mathrm{T}}$ in line 14 of check-preemption(c), with $c=w$. In order for check-preemption (c) even to be called on the node $w$, the node must have been marked with $\mathrm{M}_{\mathrm{tt}}$ in lines 10-11 of query $(x, y)$, and also with $\mathbf{M}_{\mathrm{ff}}$ in lines 12-13. Since $w$ is marked with $\mathbf{M}_{\mathrm{tt}}$, we know by an argument identical to that presented in (ii) that $\Gamma \gg x \rightarrow \sigma_{1} \rightarrow u$, that $u \rightarrow w \in \Gamma$, and that $x \nrightarrow w \notin \Gamma$. So clauses II.1(a) through II.1(c) of the inheritance definition are satisfied. Since $w$ is also marked with $\mathbf{M}_{\mathrm{ff}}$, however, an analogous argument tells us that there exist nodes $v$ such that $\Gamma \ngtr x \rightarrow \tau \rightarrow v$ and $v \rightarrow y \in \Gamma$.

If clause II.1(d) were false, there would be some such node $v$ for which there is no node $z$ such that $\Gamma \triangleright x \rightarrow \tau_{1} \rightarrow z \rightarrow \tau_{2} \rightarrow v$ and $z \rightarrow w \in \Gamma$. Let $v^{\prime}$ be a node satisfying these conditions of minimal degree ${ }^{x, y}$. Since $w$ is marked with $\mathbf{M}_{\mathrm{T}}$ on line 14 of check-preemption(c), it cannot have been marked with $\mathbf{M}_{\mathrm{ff}}$ in lines 12-13; therefore, $v^{\prime}$ must have been marked with $M_{\text {pre }}$ by the loop in lines 6-9. The effect of this loop, however, is to propagate the marker $M_{\text {pre }}$ up a positive path, all of whose nodes are marked with $M_{T}$ but not with $M_{F}$, from a node marked with $\mathrm{M}_{\mathrm{T}}$ and linked directly to $w$. Therefore, we know that by the $n$th iteration of the main loop of query $(x, y)$ there must exist a sequence of nodes $z_{0}, z_{1}, \ldots, z_{m}$ occurring in $\Gamma$ such that: each $z_{i}$ is marked with $\mathbf{M}_{\mathrm{T}}$, none of the $z_{i}$ except perhaps $z_{0}$ is marked with $\mathrm{M}_{\mathrm{F}}, z_{0} \rightarrow w \in \Gamma, z_{m}=v^{\prime}$, and $z_{i} \rightarrow z_{i+1} \in \Gamma$ for all $0 \leqslant i<m$. The inductive hypothesis tells us that, for $0 \leqslant i \leqslant m, \Gamma$ supports each of the statements $x \rightarrow z_{i}$, and also that, for $0<i \leqslant m$, none of the statements $x \rightarrow z_{i}$ is contained in $\Gamma$. We can then conclude from Lemma 6.5 that $\Gamma$ permits a path of the form $x \rightarrow \tau_{0} \rightarrow z_{0} \rightarrow z_{1} \rightarrow \cdots \rightarrow z_{n}\left(=v^{\prime}\right)$. Since $z_{0} \rightarrow w \in \Gamma$, this contradicts the above assumption concerning $v^{\prime}$; and so clause II.1(d) must be true. $\Gamma$ therefore permits the path $x \rightarrow \sigma_{1} \rightarrow u$ and so supports $x \rightarrow w$.

Part 3: Completeness for negative paths. Similar to Part 1.

Part 4: Soundness for negative paths. Similar to Part 2.

Lemma 6.5. For a sequence of nodes $z_{0}, z_{1}, \ldots, z_{m}$, suppose that

(i) $\Gamma$ supports $x \rightarrow z_{i}$ for $0 \leqslant i \leqslant m$,

(ii) $z_{i} \rightarrow z_{i+1} \in \Gamma$ for $0 \leqslant i<m$, and

(iii) $x \rightarrow z_{i} \notin \Gamma$ for $0<i \leqslant m$.

Then $\Gamma$ permits a path of the form $x \rightarrow \tau_{0} \rightarrow z_{0} \rightarrow z_{1} \rightarrow \cdots \rightarrow z_{m}$.

Proof. It follows from (i) that $\Gamma \ngtr x \rightarrow \tau_{0} \rightarrow z_{0}$, for some $\tau_{0}$. Assuming $\Gamma P x \rightarrow \tau_{0} \rightarrow z_{0} \rightarrow z_{1} \rightarrow \cdots \rightarrow z_{n}$, for some $n<m$, we show that $\Gamma \gg x \rightarrow \tau_{0} \rightarrow z_{0} \rightarrow z_{1} \rightarrow \cdots \rightarrow z_{n} \rightarrow z_{n+1}$. We satisfy clause II.1(a) by inductive hypothesis; we satisfy II.1(b) by (ii) and II.1(c) by (iii). Finally, suppose there exists $v$ such that $\Gamma \triangleright x \rightarrow \tau \rightarrow v$ with $v \rightarrow z_{n+1} \in \Gamma$. By (i) we know that $\Gamma \triangleright x \rightarrow \tau_{n+1} \rightarrow z_{n+1}$ for some path $\tau_{n+1}$. Therefore there exists $z$ such that $z \rightarrow z_{n+1} \in \Gamma$ and either $z=x$ or $\Gamma \ngtr x \rightarrow \tau^{\prime} \rightarrow z \rightarrow \tau^{\prime \prime} \rightarrow v$. So clause II.1(d) is satisfied as well. 


\subsection{Performance of the algorithm}

We now give bounds on the running time of query $(x, y)$ in a net $\Gamma$.

Let $d(x)$ be the length of the longest shortest path from $x$ to any node in $\Gamma$. The procedure trim-for-query $(x, y)$, which propagates $\mathbf{M}_{1}$ up and $\mathbf{M}_{2}$ down the hierarchy between $x$ and $y$, runs in $\mathrm{O}(d(x))$ time. Note that $d(x)$ depends on the network as a whole; it does not depend on $y$. In most practical applications, however, $d(x)$ is not expected to be significant. ${ }^{6}$

The procedure select-next-degree() contains no loops. It therefore runs in constant time.

Let $C(x, y)$ represent the number of conflicted nodes encountered when computing query $(x, y)$ in $\Gamma$. Conflicted nodes are terminal nodes of compound paths that conflict with some other compound path, but not with any direct path. That is, the conflicted nodes in $\Gamma$ are those nodes $c$ such that: (i) $\Gamma$ permits paths of the form $x \rightarrow \sigma \rightarrow u$ and $x \rightarrow \tau \rightarrow v$, where $\Gamma$ contains both the links $u \rightarrow y$ and $v \nrightarrow y$, and (ii) $\Gamma$ contains neither of the links $x \rightarrow y$ or $x \nrightarrow y$. These nodes will be marked with both $\mathrm{M}_{\mathrm{tt}}$ and $\mathrm{M}_{\mathrm{ff}}$ by query $(x, y)$; then check-preemption must be called sequentially for each node. Let $d(x, y)$ denote the length of the longest shortest path from $x$ to any other node in the trimmed network $\Gamma^{x, y}$. Obviously, $d(x, y) \leqslant d(x)$. The procedure check-preemption contains a single loop that propagates $\mathrm{M}_{\text {pre }}$ up " $\rightarrow$ " links in $\Gamma^{x, y}$, it runs in $\mathrm{O}(d(x, y))$ time.

Let $D(x, y)$ be the "depth" of the trimmed network $\Gamma^{x, y}$, that is, the degree $^{x, y}$ of the node $y$. Note that $d(x, y) \leqslant D(x, y)$. The main loop of query $(x, y)$ is executed at most $1+D(x, y)$ times. Inside this loop is the for loop that calls check-preemption $(c)$, the total number of calls to which is $C(x, y)$, each running in time $\mathrm{O}(d(x, y))$. Therefore, the running time of the entire procedure query $(x, y)$ is

$$
\mathrm{O}(\max (d(x), D(x, y)+[C(x, y) \cdot d(x, y)])) .
$$

Since $d(x)$ is not expected to be a significant factor in realistic knowledge bases, the running time of a query $(x, y)$ in such a knowledge base will be

$$
\mathrm{O}(D(x, y)+[C(x, y) \cdot d(x, y)])
$$

In a realistic knowledge base containing very few conflicted nodes, the running time of query $(x, y)$ will of course approach the purely parallel $\mathrm{O}(D(x, y))$.

\footnotetext{
${ }^{6}$ If inheritance were completely stable (see Section 5.3), the running time for trim-forquery $(x, y)$ could be incrementally reduced to constant time, or even a single time step, by modifying the query procedure so that it adds redundant links to $\Gamma$ as a side-effect until $d(x)$ falls below the desired value. Since inheritance fails to exhibit generic stability, however, we would have to resort instead to more complex conditioning procedures (mentioned below, in Section 6.5) to improve the running time of this part of the algorithm.
} 


\subsection{Discussion}

The PMPM architecture we described in this section is rather limited in computational power, which makes economical implementations possible. Fahlman estimated in [6] that a million-element marker propagation machine could be constructed using just a few custom VLSI chips, plus a lot of RAM. The connection machine [8], which is the closest physical realization to our idealized PMPM, was inspired by Fahlman's work; the algorithm we present here is thus well suited to the connection machine. Our algorithm also maps very naturally onto other parallel architectures. We briefly mention two extensions.

First, suppose the PMPM architecture is modified so that, in a network with $N$ nodes, each node has at least $2 N$ marker bits, so that the entire theory of the network can be represented at once. We reserve the bits $\mathrm{M}_{\mathrm{T} x}$ and $\mathrm{M}_{\mathrm{F} x}$ for each node $x$ in the net. If $y$ is marked with $\mathrm{M}_{\mathrm{T} x}$ it indicates that $\Gamma$ permits $x \rightarrow y$, and similarly for $\mathrm{M}_{\mathrm{F} x}$. A PMPM with this many marker bits could simply compute the entire theory of a network at once, and then use table lookup to answer queries. A more practical approach would be to compute the results of queries as needed and cache them in the nodes.

A second possible extension involves the sequential calls to check-preemption $(c)$ in the body of the for loop in the main inference procedure. Suppose that we had the ability to propagate $m$ different sets of markers independently, in parallel. (This might be the case if a PMPM were being simulated on a dataflow architecture ith $m$ processors.) We could then create $m$ sets of markers $\mathrm{M}_{c, i}, \mathrm{M}_{\mathrm{tt}, i}, \mathrm{M}_{\mathrm{ff}, i}, \mathrm{M}_{\mathrm{dir}, i}$, and $\mathrm{M}_{\mathrm{pre}, i}$, for $1 \leqslant i \leqslant m$, and process conflicted nodes of degree $i$ in parallel, $m$ at a time.

Two related inheritance reasoners that have appeared in the literature recently are Touretzky's TINA [22], and Etherington's nondeterministic algorithm [4]. Both are implementations of credulous rather than skeptical definitions. TINA (for Topological Inference Architecture) computes the credulous extension of a network according to the theory provided by [22], provided that the extension is unique. If the network has multiple credulous extensions, TINA detects this fact and issues an error message. Etherington's algorithm generates one credulous extension, but which one depends on the choice of order in which markers are propagaged; this is the source of the nondeterminism. Etherington notes that in some cases the algorithm will never choose certain extensions which his theory permits.

In addition to computing the extension, TINA had another function. In a process known as "conditioning," it augmented the inheritance network with extra links so that a marker propagation algorithm, called an upscan, could reconstruct portions of the extension as needed. The upscan algorithm was quite simple: it used shortest-path reasoning and had no sequential bottleneck, unlike the algorithm presented here. We should emphasize, however, that its 
purpose was to reconstruct an extension rather than compute it. ${ }^{7}$ The conditioning process, which takes a network and its correct extension as inputs, alters the network as necessary to ensure that upscans produce correct results. A similar trick could be used with any inheritance definition.

TINA also supported a second kind of scan, called a downscan. A downscan of $y$ marked all nodes $x$ such that $\Gamma$ permits $x \rightarrow y$ or (represented by a different marker) $x \rightarrow y$. Downscans are useful for finding all the members of a set, which can then be intersected with other sets in parallel, for example, to find all the gray elephants by intersecting elephants and gray things. It would appear to be far more expensive to compute downscans than upscans on a PMPM. Each conflicted node $c$ encountered during a downscan from $y$ would have to be resolved individually by calling query $(c, y)$.

\section{Conclusion}

We have presented in this paper a new, skeptical theory of inheritance reasoning in nonmonotonic semantic networks. As far as we know, this theory represents the first significant alternative to the analysis of nonmonotonic inheritance reasoning presented in Touretzky [22]. (A less radical alternative is described by Sandewall in [20]; although it differs in some ways from Touretzky's, Sandewall's is nevertheless a credulous theory.) The fact that there should be distinct but, perhaps, equally well-motivated accounts of correct reasoning in this context comes as something of a surprise; it is reminiscent of the situation in philosophical logic, where there exist rival logics embodying distinct conceptions of correct deductive reasoning.

In the context of inheritance reasoning, the existence of these distinct approaches raises a number of issues, which we are exploring in our current research. Much of this research is focused more or less directly on inheritance theory: we are studying the relations among the different analyses of nonmonotonic inheritance reasoning $[9,23,24]$ and working to extend some of these analyses to more expressive nonmonotonic network languages [10,25]. However, it is also possible that this research will shed some light on more general treatments of nonmonotonic reasoning. It has been shown by Etherington [4], for example, that the default logic of Reiter [18] can be used to provide a specification for correct inheritance reasoning in nonmonotonic semantic networks: Etherington establishes a close correspondence between these networks and certain kinds of default theories ("network default theories"). But these results, linking default logic to nonmonotonic inheri-

\footnotetext{
${ }^{7}$ It does not appear possible to compute extensions on a PMPM according to the inheritance definition provided in [22], even when the extension is unique. The difference appears to be unrelated to the choice between the skeptical and credulous approaches. Instead, it derives from the fact that [22] relies on a slightly different treatment of preemption, which forces a reasoning architecture to pay more attention to actual paths, rather than just supported conclusions. We compare these two styles of preemption in [23].
} 
tance, presuppose a credulous analysis of inheritance reasoning; this bias toward the credulous approach to nonmonotonic reasoning is in fact built into Reiter's default logic, and also into the nonmonotonic logics of McDermott and Doyle [13] and Moore [14]. Since, as we have shown, there turns out to be an equally well-motivated skeptical theory of nonmonotonic reasoning, at least in the case of semantic networks, it might be useful at this point to seek a weaker version of default or nonmonotonic logic, exhibitiong instead a bias toward skepticism-or perhaps a more general logic that is neutral between the credulous and skeptical approaches.

\section{ACKNOWLEDGEMENT}

This material is based on work supported by the National Science Foundation under Grants No. IST-8516313 and IRI-8700705.

We are grateful to Charles Cross for many valuable discussions during the preparation of this paper, and to David Makinson for filling a gap in one of our proofs.

\section{REFERENCES}

1. N. Belnap, How a computer should think, in: G. Ryle, ed., Contemporary Aspects of Philosophy (Oriel Press, 1977) 30-56.

2. N. Belnap, N., A useful four-valued logic, in: J. Dunn and G. Epstein, eds., Modern Uses of Multiple-valued Logic (Reidel, Dordrecht, Netherlands, 1977) 8-37.

3. G. Carlson, Generic terms and generic sentences, J. Philos. Logic 11 (1982) 145-181.

4. D. Etherington, Formalizing nonmonotonic reasoning systems, Artificial Intelligence 31 (1987) 41-85.

5. S. Fahlman, NETL: A System for Representing and Using Real-World Knowledge (MIT Press, Cambridge, MA, 1979).

6. S. Fahlman, Design sketch for a million-element NETL machine, in: Proceedings AAAI-80, Stanford, CA (1980) 249-252.

7. S. Fahlman, D. Touretzky and W. van Roggen, Cancellation in a parallel semantic network, in: Proceedings IJCAI-81, Vancouver, BC (1981) 257-263.

8. W. Hillis, The Connection Machine (MIT Press, Cambridge, MA, 1985).

9. J. Horty, Some direct theories of nonmonotonic inheritance. Tech. Rept., Institute for Advanced Computer Studies, University of Maryland, College Park, MD (1990).

10. J. Horty and R. Thomason, Mixing strict and defeasible inheritance, in: Proceedings AAAI88, St. Paul, MN (1988) 427-432.

11. J. Horty, R. Thomason and D. Touretzky, A skeptical theory of inheritance in nonmonotonic semantic networks, Tech. Rept. CMU-CS-87-175, Computer Science Department, CarnegieMellon University, Pittsburgh, PA (1987).

12. J. McCarthy, Circumscription: A form of non-monotonic reasoning, Artificial Intelligence $\mathbf{1 3}$ (1980) 27-39.

13. D. McDermott and J. Doyle, Non-monotonic logic I, Artificial Intelligence 13 (1980) 41-72.

14. R. Moore, Semantical considerations on nonmonotonic logic, Artificial Intelligence 25 (1985) 75-94.

15. R. Nado and R. Fikes, Semantically sound inheritance for a formally defined frame language with defaults, in: Proceedings AAAI-87, Philadelphia, PA (1987) 443-448.

16. M. Quillian, Semantic memory, Ph.D. Dissertation, Carnegie Institute of Technology, Pittsburgh, PA (1966); also in: M. Minsky, ed., Sernantic Information Processing (MIT Press, Cambridge, MA, 1968). 
17. A. Rector, Defaults, exceptions and ambiguity in a medical knowledge representation system, Med. Inf. 11 (1986) 295-306.

18. R. Reiter, A logic for default reasoning, Artificial Intelligence 13 (1980) 81-132.

19. R. Roberts and I. Goldstein, The FRL manual, AI Memo No. 409, Artificial Intelligence Laboratory, MIT, Cambridge, MA (1977).

20. E. Sandewall, Non-monotonic inference rules for multiple inheritance with exceptions, Proc. IEEE 74 (1986) 1345-1353.

21. R. Thomason, J. Horty and D. Touretzky, A calculus for inheritance in monotonic semantic nets, Tech. Rept. CMU-CS-86-138, Computer Science Department, Carnegie-Mellon University, Pittsburgh, PA (1986).

22. D. Touretzky, The Mathematics of Inheritance Systems (Morgan Kaufmann, Los Altos, CA, 1986).

23. D. Touretzky, J. Horty and R. Thomason, A clash of intuitions: the current state of nonmonotonic multiple inheritance systems, in: Proceedings IJCAI-87, Milan (1987) 476-482.

24. D. Touretzky, J. Horty and R. Thomason, Issues in the design of nonmonotonic inheritance systems, Tech. Rept., Computer Science Department, Carnegie-Mellon University, Pittsburgh, PA (1990).

25. D. Touretzky and R. Thomason, Nonmonotonic inheritance and generic reflexives, in: Proceedings AAAI-88, St. Paul, MN (1988) 433-438.

Received November 1987; revised version received August 1988/November 1988 